\title{
Magnetosphere-ionosphere coupling currents in Jupiter's middle magnetosphere: dependence on the effective ionospheric Pedersen conductivity and iogenic plasma mass outflow rate
}

\author{
J. D. Nichols ${ }^{1}$ and S. W. H. Cowley ${ }^{1}$ \\ ${ }^{1}$ Department of Physics and Astronomy, University of Leicester, Leicester LE1 7RH, UK
}

Received: 27 August 2002 - Revised: 9 December 2002 - Accepted: 20 February 2003

\begin{abstract}
The amplitude and spatial distribution of the coupling currents that flow between Jupiter's ionosphere and middle magnetosphere, which enforce partial corotation on outward-flowing iogenic plasma, depend on the values of the effective Pedersen conductivity of the jovian ionosphere and the mass outflow rate of iogenic plasma. The values of these parameters are, however, very uncertain. Here we determine how the solutions for the plasma angular velocity and current components depend on these parameters over wide ranges. We consider two models of the poloidal magnetospheric magnetic field, namely the planetary dipole alone, and an empirical current sheet field based on Voyager data. Following work by Hill (2001), we obtain a complete normalized analytic solution for the dipole field, which shows in compact form how the plasma angular velocity and current components scale in space and in amplitude with the system parameters in this case. We then obtain an approximate analytic solution in similar form for a current sheet field in which the equatorial field strength varies with radial distance as a power law. A key feature of the model is that the current sheet field lines map to a narrow latitudinal strip in the ionosphere, at $\approx 15^{\circ}$ co-latitude. The approximate current sheet solutions are compared with the results of numerical integrations using the full field model, for which a power law applies beyond $\approx 20 R_{J}$, and are found to agree very well within their regime of applicability. A major distinction between the solutions for the dipole field and the current sheet concerns the behaviour of the field-aligned current. In the dipole model the direction of the current reverses at moderate equatorial distances, and the current system wholly closes if the model is extended to infinity in the equatorial plane and to the pole in the ionosphere. In the approximate current sheet model, however, the field-aligned current is unidirectional, flowing consistently from the ionosphere to the current sheet for the sense of the jovian magnetic field. Current closure must then occur at higher latitudes, on field lines outside the region described by the model. The amplitudes
\end{abstract}

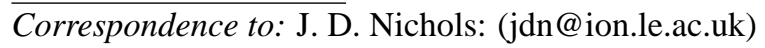

of the currents in the two models are found to scale with the system parameters in similar ways, though the scaling is with a somewhat higher power of the conductivity for the current sheet model than for the dipole, and with a somewhat lower power of the plasma mass outflow rate. The absolute values of the currents are also higher for the current sheet model than for the dipole for given parameters, by factors of $\approx 4$ for the field-perpendicular current intensities, $\approx 10$ for the total current flowing in the circuit, and $\approx 25$ for the field-aligned current densities, factors which do not vary greatly with the system parameters. These results thus confirm that the conclusions drawn previously from a small number of numerical integrations using spot values of the system parameters are generally valid over wide ranges of the parameter values.

Key words. Magnetospheric physics (current systems, magnetosphere-ionosphere interactions, planetary magnetospheres)

\section{Introduction}

The dynamics of Jupiter's middle magnetosphere are dominated by the processes that couple angular momentum between the planet's atmosphere and the equatorial plasma that flows outwards from the Io source at $\approx 6 \mathrm{R}_{\mathrm{J}}$ (Jupiter's radius, $R_{J}$, is $\approx 71373 \mathrm{~km}$ ) (Hill, 1979; Siscoe and Summers, 1981; Hill et al., 1983; Belcher, 1983; Vasyliunas, 1983; Bagenal, 1994). The field and plasma structures envisaged are sketched in Fig. 1, where the arrowed solid lines indicate magnetic field lines, while the dots indicate the region occupied by dense rotating iogenic plasma. The region of flux tubes threading this plasma disc constitutes Jupiter's middle magnetosphere, where the field lines are characteristically distended outward by azimuthal plasma currents associated with radial stress balance. In the inner region, the iogenic plasma approximately corotates with the planet, but as it moves outward its angular velocity falls, as the inverse square of the distance if no torques act. However, when the angular 


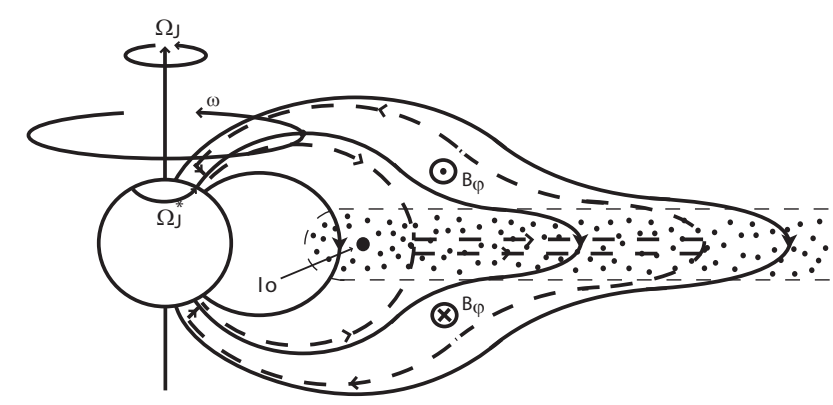

Fig. 1. Sketch of a meridian cross section through Jupiter's inner and middle magnetosphere, showing the principal physical features involved. The arrowed solid lines indicate magnetic field lines, the arrowed dashed lines the magnetosphere-ionosphere coupling current system, and the dotted region the rotating disc of out-flowing iogenic plasma. (From Cowley and Bunce, 2001).

velocity of the plasma and field lines ( $\omega$ in Fig. 1) falls below that of the planet $\left(\Omega_{J}\right)$, or more specifically, below that of the neutral upper atmosphere $\left(\Omega_{J}^{*}\right)$, a frictional torque is imposed on the feet of the field lines due to ion-neutral collisions in the Pedersen-conducting layer of the ionosphere. This torque acts to spin the flux tubes and equatorial plasma back up towards rigid corotation with the planet, so that in the steady state the plasma angular velocity falls less quickly with distance than as the inverse square. At the same time, the equal and opposite torque on the neutral atmosphere results in atmospheric sub-corotation ("slippage") in the Pedersen layer, so that $\omega<\Omega_{J}^{*}<\Omega_{J}$ (Huang and Hill, 1989). The spin-up torque on the plasma is communicated to the equatorial region by the magnetic field, which becomes bent out of meridian planes into a "lagging" configuration, associated with the azimuthal fields $B_{\varphi}$ shown in the figure. The corresponding magnetosphere-ionosphere coupling current system, of primary interest here, is shown by the arrowed dashed lines in Fig. 1. The current flows radially outward across the field in the equatorial plane, such that the torque associated with the $\boldsymbol{j} \times \boldsymbol{B}$ force accelerates the plasma in the sense of Jupiter's rotation. In the ionosphere the Pedersen current flows equatorward in both hemispheres, producing an equal and opposite torque which balances the torque due to ionneutral collisions. The current circuit is closed by a system of field-aligned currents which flow from the ionosphere to the equator in the inner part of the system, and return in the outer part.

The steady-state angular velocity profile of the out-flowing equatorial plasma was first calculated on the above basis by Hill (1979). In this study the poloidal field was taken to be that of the planetary dipole alone, such that the radial distension of the middle magnetosphere field lines shown in Fig. 1 was not taken into account. This restriction was later removed by Pontius (1997), who introduced empirical poloidal field models into the calculations. He found that the solutions for the plasma angular velocity profile are remarkably insensitive to the field model employed. Although consid- ered implicitly, the properties of the coupling currents were not calculated in these studies. Recently, however, attention has focussed directly on the currents, it being suggested by a number of authors that the ring of upward field-aligned current surrounding each magnetic pole is associated with the "main oval" observed in Jupiter's auroras (Bunce and Cowley, 2001; Cowley and Bunce, 2001; Hill, 2001; Southwood and Kivelson, 2001). Hill (2001) considered the currents in his original dipole problem, while Cowley and Bunce (2001) calculated the currents for both a dipole field and an empirical current sheet field. The latter authors found that the fieldaligned current densities are an order of magnitude larger for the current sheet model than for the dipole. The physical origins of this effect have been discussed further by Cowley et al. $(2002,2003)$, who show that it relates to the fact that the field lines on which corotation breaks down, while mapping to similar distances in the equatorial plane, map in the ionosphere to a narrower range of latitudes further from the pole for a current sheet field than for the dipole.

The solutions for the plasma angular velocity and the current depend on two system parameters, the "effective" value of the height-integrated ionospheric Pedersen conductivity (possibly reduced from the true value by the atmospheric "slippage" mentioned above), and the plasma mass outflow rate from the Io torus. However, neither of these parameters is well determined at present. Estimates of the conductivity have ranged from $\approx 0.1$ to $\approx 10$ mho (Strobel and Atreya, 1983; Bunce and Cowley, 2001), while estimates of the mass outflow rate have ranged from $\approx 500$ to $\approx 2000 \mathrm{~kg} \mathrm{~s}^{-1}$ (Broadfoot et al., 1981; Hill et al., 1983; Vasyliunas, 1983; Khurana and Kivelson, 1993; Bagenal, 1997). The purpose of the present paper is to examine how the solutions for the plasma angular velocity and coupling currents depend on these two parameters for both dipole and current sheet field models. Some general results for the dipole model have been presented previously by Hill (2001). For the current sheet field model, however, only a few numerical solutions using "reasonable" spot values of the system parameters have been published to date (Cowley and Bunce, 2001; Cowley et al., 2002, 2003). In this paper we first provide a complete solution for the dipole field, before going on to examine related results for a current sheet model in which the equatorial field is taken to vary with distance as a power law. The parameter ranges considered are $0.1-10$ mho for the "effective" conductivity, and $100-10000 \mathrm{~kg} \mathrm{~s}^{-1}$ for the mass outflow rate. The work presented here thus shows how the coupling current system depends on the system parameters over a wide range of values, here taken as constant quantities in a given solution. This knowledge should provide valuable background for more complex future studies in which the system parameters are taken to vary in time and/or space, as may more generally be the case. 


\section{Basic equations}

In this section we first summarise the basic equations governing the magnetosphere-ionosphere coupling current system depicted in Fig. 1, and then discuss the nature of the solutions at small and large distances. Derivations were given earlier by Hill (1979), Vasyliunas (1983) and Pontius (1997), and have most recently been discussed by Hill (2001), Cowley and Bunce (2001), and Cowley et al. (2003). Only the central results will, therefore, be stated here, together with an outline of the assumptions and approximations which have been made.

We first assume that the magnetic field is axisymmetric, such that the poloidal components can be specified by a flux function $F(\rho, z)$ related to the field components by $\boldsymbol{B}=(1 / \rho) \nabla F \times \hat{\varphi}$, where $\rho$ is the perpendicular distance from the magnetic axis, $z$ is the distance along this axis from the magnetic equator, and $\varphi$ is the azimuthal angle. In this case $F=$ constant defines a flux shell, such that magnetic mapping between the equatorial plane (subscript " $e$ ") and the ionosphere (subscript " $i$ "), as required here, is simply achieved by writing $F_{e}=F_{i}$. Neglecting non-dipole planetary fields and the small perturbations due to magnetospheric currents, the flux function in the ionosphere is taken to be

$F_{i}=B_{J} \rho_{i}^{2}=B_{J} R_{J}^{2} \sin ^{2} \theta_{i}$,

where $\rho_{i}$ is the perpendicular distance from the magnetic axis, $\theta_{i}$ the magnetic co-latitude, and $B_{J}$ the dipole equatorial magnetic field strength (taken to be $4.28 \times 10^{5} \mathrm{nT}$ in conformity with the VIP 4 internal field model of Connerney et al., 1998). The absolute value of $F$ has been fixed by taking $F=0$ on the magnetic axis. The flux function in the equatorial plane is then obtained by integrating

$B_{z e}=\frac{1}{\rho_{e}} \frac{d F_{e}}{d \rho_{e}}$,

where $B_{z e}\left(\rho_{e}\right)$ is the north-south field threading the current sheet.

Assuming for simplicity that the magnetic and planetary spin axes are co-aligned, the equatorward-directed heightintegrated Pedersen current in the ionosphere is given by

$$
\begin{aligned}
i_{P} & =2 \Sigma_{P}^{*} B_{J} \Omega_{J} \rho_{i}\left(1-\frac{\omega}{\Omega_{J}}\right) \\
& =2 \Sigma_{P}^{*} B_{J} \Omega_{J} \sqrt{\frac{F_{i}}{B_{J}}}\left(1-\frac{\omega}{\Omega_{J}}\right),
\end{aligned}
$$

where we have taken the polar magnetic field to be nearvertical and equal to $2 B_{J}$ in strength (an approximation valid to within $\approx 5 \%$ in our region of interest). In this expression, $\Sigma_{p}^{*}$ is the "effective" height-integrated ionospheric Pedersen conductivity, reduced from the true value $\Sigma_{p}=\Sigma_{p}^{*} /(1-k)$ by neutral atmosphere "slippage" as mentioned above, where parameter $k$ (whose value $0<k<1$ is also uncertain at present) is related to the angular velocities by $\left(\Omega_{J}-\Omega_{J}^{*}\right)=$
$k\left(\Omega_{J}-\omega\right)$ (Huang and Hill, 1989). The radial current intensity in the equatorial plane $i_{\rho}$, integrated through the full thickness of the sheet, then follows from the current continuity requirement $\rho_{e} i_{\rho}=2 \rho_{i} i_{P}$ (assuming symmetry between the two hemispheres)

$i_{\rho}=\frac{4 \Sigma_{P}^{*} \Omega_{J} F_{e}}{\rho_{e}}\left(1-\frac{\omega}{\Omega_{J}}\right)$,

so that the total equatorial radial current, integrated in azimuth, is

$I_{\rho}=2 \pi \rho_{e} i_{\rho}=8 \pi \Sigma_{P}^{*} \Omega_{J} F_{e}\left(1-\frac{\omega}{\Omega_{J}}\right)$,

equal, of course, to twice the azimuth-integrated total Pedersen current $I_{P}=2 \pi \rho_{i} i_{P}$ flowing in each conjugate ionosphere. The field-aligned current density follows from the divergence of either of these field-perpendicular currents. Differentiating the equatorial current gives

$$
\begin{aligned}
& \left(\frac{j_{\|}}{B}\right)=\frac{1}{4 \pi \rho_{e}\left|B_{z e}\right|} \frac{d I_{\rho}}{d \rho_{e}}= \\
& \quad-2 \Sigma_{P}^{*} \Omega_{J}\left(\left(\frac{F_{e}}{\rho_{e} \mid B_{z e}}\right) \frac{d}{d \rho_{e}}\left(\frac{\omega}{\Omega_{J}}\right)+\left(1-\frac{\omega}{\Omega_{J}}\right)\right),
\end{aligned}
$$

where we have put $B_{z e}=-\left|B_{z e}\right|$, since the jovian field is negative (points south) at the equator, $\Sigma_{P}^{*}$ has been taken to be constant, and the sign of $j_{\|}$is appropriate to the Northern Hemisphere (as employed throughout). The quantity $\left(j_{\|} / B\right)$ is constant along field lines between the equator and the ionosphere in the assumed absence of significant fieldperpendicular currents in the intervening region. The fieldaligned current density just above the ionosphere is then given by

$j_{\| i}=2 B_{J}\left(\frac{j_{\|}}{B}\right)$,

using the same approximation for the polar field as indicated above.

The analysis is completed by determining the steady state angular velocity profile of the equatorial plasma. Following Hill (1979, 2001), Pontius (1997), and Cowley et al. (2002), Newton's second law applied to a steady outflow of plasma from the Io torus gives

$$
\frac{d}{d \rho_{e}}\left(\rho_{e}^{2} \omega\left(\rho_{e}\right)\right)=\frac{2 \pi \rho_{e}^{2} i_{\rho}\left|B_{z e}\right|}{\dot{M}},
$$

where $\dot{M}$ is the plasma mass outflow rate. Expanding and substituting Eq. (4a), we find

$$
\frac{\rho_{e}}{2} \frac{d}{d \rho_{e}}\left(\frac{\omega}{\Omega_{J}}\right)+\left(\frac{\omega}{\Omega_{J}}\right)=\frac{4 \pi \Sigma_{P}^{*} F_{e}\left|B_{z e}\right|}{\dot{M}}\left(1-\frac{\omega}{\Omega_{J}}\right),
$$

an equation we refer to as the Hill-Pontius equation (though here slightly simplified, as in Hill (2001), by taking the ionospheric field strength to be equal to $2 B_{J}$ ). It is a first order linear equation for $\omega$ that can be solved with the use of one boundary condition. We note with Hill (2001) that if the angular velocity profile obeys this equation, the derivative may 
be substituted directly into Eq. (5a) to yield a form for the field-aligned current which involves $\left(\omega / \Omega_{J}\right)$ only.

It is an important general property of the physically interesting solutions of the above equations that at small radial distances the currents depend on $\dot{M}$ and not on $\Sigma_{P}^{*}$, while at large radial distances they depend on $\Sigma_{P}^{*}$ and not on $\dot{M}$. The small- $\rho_{e}$ approximations follow from a series solution of Eq. (6b) for the case in which $\left(\omega / \Omega_{J}\right)=1$ at $\rho_{e}=0$ (such that the plasma rigidly corotates at small distances). Taking $\left(\dot{M} / \Sigma_{P}^{*}\right)$ as the formal expansion parameter, we write

$$
\frac{\omega}{\Omega_{J}}=\sum_{n=0}^{\infty} a_{n}\left(\frac{\dot{M}}{\Sigma_{P}^{*}}\right)^{n}
$$

and substituting into Eq. (6b) and equating coefficients of powers of $\left(\dot{M} / \Sigma_{P}^{*}\right)$ we find that $a_{0}=1$, and that for $n \geq 1$ the $a_{n}$ are determined by the recurrence relation

$a_{n+1}=-\frac{1}{8 \pi \rho_{e} F_{e}\left|B_{z e}\right|} \frac{d}{d \rho_{e}}\left(\rho_{e}^{2} a_{n}\right)$.

Thus, the leading term describing the breakdown of rigid corotation in the inner region which we take as our small$\rho_{e}\left({ }^{\prime} S '\right)$ approximation is

$$
\left(\frac{\omega}{\Omega_{J}}\right)_{S}=1-\frac{\dot{M}}{4 \pi \Sigma_{P}^{*} F_{e}\left|B_{z e}\right|},
$$

as given previously (but not derived in this manner) by Cowley et al. (2003). We note that the departure from rigid corotation is proportional to $\dot{M}$ and inversely proportional to $\Sigma_{P}^{*}$. When substituted into Eqs. (3)-(5) to find the corresponding approximations for the currents, we then find that the currents on a given field line depend only on $\dot{M}$ and not on $\Sigma_{P}^{*}$

$i_{P S}=\frac{\dot{M} \Omega_{J}}{2 \pi \rho_{i}\left|B_{z e}\right|}, \quad I_{P S}=\frac{\dot{M} \Omega_{J}}{\left|B_{z e}\right|}$,

$i_{\rho S}=\frac{\dot{M} \Omega_{J}}{\pi \rho_{e}\left|B_{z e}\right|}, \quad I_{\rho S}=\frac{2 \dot{M} \Omega_{J}}{\left|B_{z e}\right|}$,

$\left(\frac{j_{\|}}{B}\right)_{S}=-\frac{\dot{M} \Omega_{J}}{2 \pi \rho_{e}\left|B_{z e}\right|^{3}} \frac{d\left|B_{z e}\right|}{d \rho_{e}}$,

and

$j_{\| i S}=-\frac{\dot{M} \Omega_{J} B_{J}}{\pi \rho_{e}\left|B_{z e}\right|^{3}} \frac{d\left|B_{z e}\right|}{d \rho_{e}}$.

These expressions can also be derived directly by substituting $\omega=\Omega_{J}$ into the left side of Eq. (6a), i.e. they are just the currents required to maintain near-rigid corotation in the inner region.

The large- $\rho_{e}($ ' $L$ ') approximations are simply obtained by putting $\left(\omega / \Omega_{J}\right)_{L}=0$ into Eqs. (3)-(5) to find

$i_{P L}=2 \Sigma_{P}^{*} B_{J} \Omega_{J} \rho_{i}$,

$I_{P L}=4 \pi \Sigma_{P}^{*} B_{J} \Omega_{J} \rho_{i}^{2}$, $i_{\rho L}=\frac{4 \Sigma_{P}^{*} \Omega_{J} F_{e}}{\rho_{e}}$

$I_{\rho L}=8 \pi \Sigma_{P}^{*} \Omega_{J} F_{e}$,

$\left(\frac{j_{\|}}{B}\right)_{L}=-2 \Sigma_{P}^{*} \Omega_{J}, \quad$ and

$j_{\| i L}=-4 \Sigma_{P}^{*} B_{J} \Omega_{J}$,

which thus depend only on $\Sigma_{P}^{*}$ and not on $\dot{M}$.

\section{Plasma angular velocity and coupling current system for a dipole magnetic field model}

Following the earlier work of Hill $(1979,2001)$ and Cowley and Bunce $(2001,2003)$, in this section we provide a complete analytic solution for the case where the poloidal field is taken to be the planetary dipole alone, showing how the solutions for the angular velocity and current components scale in space and in amplitude with the system parameters. These results form a useful introduction to, and point of comparison with, the results for the current sheet field to be presented in the Sect. 4. Using Eq. (2), for the dipole field we have

$B_{z e d i p}=-B_{J}\left(\frac{R_{J}}{\rho_{e}}\right)^{3}$ and hence

$F_{e d i p}=\frac{B_{J} R_{J}^{3}}{\rho_{e}}$.

Substituting these into Eq. (6b), the Hill-Pontius equation for the dipole field is

$\frac{\rho_{e}}{2} \frac{d}{d \rho_{e}}\left(\frac{\omega}{\Omega_{J}}\right)+\left(\frac{\omega}{\Omega_{J}}\right)=2\left(\frac{R_{D e}}{\rho_{e}}\right)^{4}\left(1-\frac{\omega}{\Omega_{J}}\right)$,

where $R_{D e}$ is the equatorial "Hill distance" for the dipole field (subscript ' $D$ '), given by

$\frac{R_{D e}}{R_{J}}=\left(\frac{2 \pi \Sigma_{P}^{*} B_{J}^{2} R_{J}^{2}}{\dot{M}}\right)^{1 / 4}$.

It can thus be seen that the angular velocity in this case is a function only of $\left(\rho_{e} / R_{D e}\right)$, so that the solutions scale with equatorial distance as $R_{D e}$ and hence with the system parameters as $\left(\Sigma_{P}^{*} / \dot{M}\right)^{1 / 4}$. The general solution of Eq. (12) can be obtained by the integration factor method (Hill, 1979)

$$
\begin{aligned}
& \left(\frac{\omega}{\Omega_{J}}\right)=\sqrt{\pi}\left(\frac{R_{D e}}{\rho_{e}}\right)^{2} \exp \left[\left(\frac{R_{D e}}{\rho_{e}}\right)^{4}\right] \times \\
& \times\left[\operatorname{erfc}\left[\left(\frac{R_{D e}}{\rho_{e}}\right)^{2}\right]+K\right],
\end{aligned}
$$

where $\operatorname{erfc}(z)$ is the complementary error function (related to the error function $\operatorname{erf}(z)$ by $\operatorname{erfc}(z)=1-\operatorname{erf}(z))$, and $K$ is a constant of integration. All solutions diverge at the origin except the solution with $K=0$. This special solution rigidly corotates (i.e. $\left.\left(\omega / \Omega_{J}\right)=1\right)$ when $\left(\rho_{e} / R_{D e}\right)$ goes to zero, 
and is the solution derived previously by Hill $(1979,2001)$. Mapping to the ionosphere is achieved by equating the flux functions given by Eq. (1) and (11b), such that

$\sin \theta_{i}=\frac{\rho_{i}}{R_{J}}=\sqrt{\frac{R_{J}}{\rho_{e}}}$.

Introducing an ionospheric counterpart of the "Hill distance" given by

$R_{D i}=\sqrt{\frac{R_{J}}{R_{D e}}} R_{J}=\left(\frac{\dot{M}}{2 \pi \Sigma_{P}^{*} B_{J}^{2} R_{J}^{2}}\right)^{1 / 8} R_{J}$,

we then find that the angular velocity mapped to the ionosphere is a function only of $\left(\rho_{i} / R_{D i}\right)$, where

$\left(\frac{\rho_{i}}{R_{D i}}\right)=\sqrt{\frac{R_{D e}}{\rho_{e}}}$

such that the solutions scale with distance from the magnetic pole as $\left(\dot{M} / \Sigma_{P}^{*}\right)^{1 / 8}$. With regard to physical units, introduction of the constant quantities given above yields the following values for the equatorial and ionospheric scale lengths

$\frac{R_{D e}}{R_{J}} \approx 49.21\left(\frac{\Sigma_{P}^{*}(\mathrm{mho})}{\dot{M}\left(10^{3} \mathrm{~kg} \mathrm{~s}^{-1}\right)}\right)^{1 / 4}$

and

$\frac{R_{D i}}{R_{J}} \approx 0.1426\left(\frac{\dot{M}\left(10^{3} \mathrm{~kg} \mathrm{~s}^{-1}\right)}{\Sigma_{P}^{*}(\mathrm{mho})}\right)^{1 / 8}$,

such that for system parameters at the centre of the ranges mentioned in the Introduction, i.e. $\Sigma_{P}^{*}=1$ mho and $\dot{M}=$ $1000 \mathrm{~kg} \mathrm{~s}^{-1}$, we find $R_{D e} \approx 49.2 R_{J}$ and $R_{D i} \approx 0.14 R_{J}$ (corresponding to a co-latitude of $8.2^{\circ}$ ). If we fix $\dot{M}$ at this value and allow $\Sigma_{P}^{*}$ to increase from 0.1 to $10 \mathrm{mho}$, we find that $R_{D e}$ increases from 27.7 to $87.5 \mathrm{R}$, while $R_{D i}$ decreases from 0.19 to $0.11 \mathrm{R}_{\mathrm{J}}$ (co-latitudes between $11.0^{\circ}$ and $6.1^{\circ}$ ). Similarly, if we fix $\Sigma_{P}^{*}$ at $1 \mathrm{mho}$ and allow $\dot{M}$ to increase from 100 to $10000 \mathrm{~kg} \mathrm{~s}^{-1}$, we find that $R_{D e}$ and $R_{D i}$ vary over the same ranges but in the reversed sense. Thus because $R_{D e}$ and $R_{D i}$ depend on the system parameters only as the quarter and eighth powers, respectively, they change only by modest factors as the system parameters vary widely. We note that the equatorial scales are comparable to the radial scale of the jovian middle magnetosphere, which extends to distances between $\sim 40$ and $\sim 100 \mathrm{R}_{\mathrm{J}}$, depending on local time and the state of the magnetosphere.

The solid lines in Figs. 2a and b show the normalised angular velocity solution with $K=0$ plotted versus $\left(\rho_{e} / R_{D e}\right)$ in the equatorial plane, and versus $\left(\rho_{i} / R_{D i}\right)$ in the ionosphere, respectively. Near-rigid corotation is maintained to $\left(\rho_{e} / R_{D e}\right) \approx 0.5$, beyond which $\left(\omega / \Omega_{J}\right)$ decreases rapidly, reaching 0.5 when $\left(\rho_{e} / R_{D e}\right) \approx 1.52$. We also note that solutions started with non-zero $K$ within $\left(\rho_{e} / R_{D e}\right) \leq 0.5$ converge very rapidly onto this solution at larger distances, such that the solutions are only weakly dependent on the choice
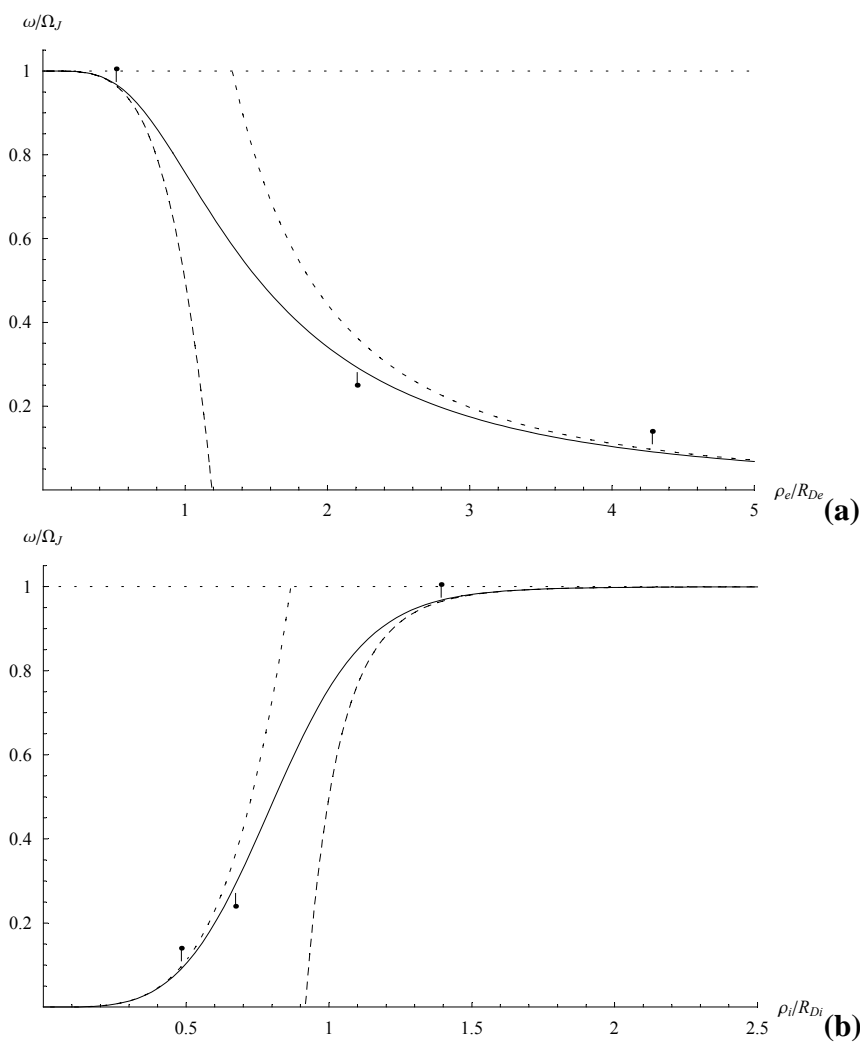

Fig. 2. Plots of the steady-state plasma angular velocity profile for a dipole magnetic field, shown (a) versus normalised radial distance in the equatorial plane $\left(\rho_{e} / R_{D e}\right)$, and (b) versus normalised distance from the magnetic axis in the ionosphere $\left(\rho_{i} / R_{D i}\right)$. The solid line in each case shows the full solution obtained from Eq. (14) with $K=0$, such that the plasma rigidly corotates at small radial distances. The long-dashed lines show the small $\rho_{e}$ form given by Eq. (19), while the corresponding large $\rho_{e}$ form is just $\left(\omega / \Omega_{J}\right)_{L}=0$. The downward-pointing tick marks indicate the limits of validity of both these approximations, as defined in the text. The short-dashed lines show the higher-order large $\rho_{e}$ form given by Eq. (20), whose limit of validity (as also defined in the text) is indicated by the upward-pointing tick mark. The horizontal dotted lines indicate the condition for rigid corotation, $\left(\omega / \Omega_{J}\right)=1$.

of boundary condition in this case (Cowley et al., 2003). The dashed lines in Fig. 2 show some approximate forms, with the tick marks indicating their regimes of validity. The longdashed lines show the small- $\rho_{e}$ (' $S$ ') approximation given by Eq. (8), which in normalised form becomes

$\left(\frac{\omega}{\Omega_{J}}\right)_{S}=1-\frac{1}{2}\left(\frac{\rho_{e}}{R_{D e}}\right)^{4}=1-\frac{1}{2}\left(\frac{R_{D i}}{\rho_{i}}\right)^{8}$.

(We note that the series generated by Eq. (7) is the same as that obtained by asymptotic expansion of the error function in Eq. (14) for large argument.) The approximate form falls away from rigid corotation more quickly than the full solution, and reaches zero, equal to the large- $\rho_{e}\left({ }^{\prime} L^{\prime}\right)$ approximation $\left(\omega / \Omega_{J}\right)_{L}=0$ at $\left(\rho_{e} / R_{D e}\right)=\sqrt[4]{2} \sim 1.189$. We define 
Table 1. Principal features of the plasma angular velocity and coupling current system for a dipole field in normalised units

\begin{tabular}{|c|c|c|c|}
\hline Feature & $\left(\frac{\rho_{e}}{R_{D e}}\right)$ & $\left(\frac{\rho_{i}}{R_{D i}}\right)$ & $\left.\frac{\omega}{\Omega_{J}}\right)$ \\
\hline $\begin{array}{l}\text { Maximum upward field-aligned current density } \\
\left(\left(j_{\|} / B\right) /\left(j_{\|} / B\right)_{D}\right)_{\text {max }}=\left(j_{\| i} / j_{\| i D}\right)_{\max } \approx 0.6111\end{array}$ & 1.0203 & 0.9900 & 0.7467 \\
\hline $\begin{array}{l}\text { Maximum sheet-integrated equatorial radial current } \\
\left(i_{\rho} / i_{\rho D}\right)_{\text {max }} \approx 0.9809\end{array}$ & 1.1034 & 0.9520 & 0.7014 \\
\hline Plasma angular velocity falls to $\left(\frac{\omega}{\Omega_{J}}\right)=0.5$ & 1.5201 & 0.8111 & 0.5 \\
\hline $\begin{array}{l}\text { Maximum azimuth-integrated total current } \\
\left(I_{\rho} / I_{\rho D}\right)_{\text {max }}=2\left(I_{P} / I_{P D}\right)_{\max } \approx 8.404 \\
\text { Field-aligned current passes through zero }\end{array}$ & 1.7409 & 0.7579 & 0.4178 \\
\hline $\begin{array}{l}\text { Maximum height-integrated ionospheric Pedersen } \\
\text { current }\left(i_{P} / i_{P D}\right)_{\max } \approx 0.9631\end{array}$ & 2.5674 & 0.6241 & 0.2284 \\
\hline $\begin{array}{l}\text { Maximum downward field-aligned current density } \\
\left(\left(j_{\|} / B\right) /\left(j_{\|} / B\right)_{D}\right)_{\min }=\left(j_{\| i} / j_{\| i D}\right)_{\min }=-2\end{array}$ & $\infty$ & 0 & 0 \\
\hline
\end{tabular}

the limits of validity of these approximations as being the points where $\left(1-\left(\omega / \Omega_{J}\right)_{S, L}\right)=1.1\left(1-\left(\omega / \Omega_{J}\right)\right)$, such that the departure from rigid corotation given by the approximate form exceeds that of the full solution by $10 \%$ of the latter. These limiting positions are shown by the downwardpointing tick marks in Fig. 2. The short-dashed lines in the figure also show a higher-order large- $\rho_{e}$ form (' $L$ ') $)$, in which the plasma angular velocity falls with distance as $\rho_{e}^{-2}$, due to negligible ionospheric torque. Noting that both the exponential and the error functions go to unity in Eq. (14) as $\rho_{e} \rightarrow \infty$, we find with Hill (1979) that for $K=0$

$$
\left(\frac{\omega}{\Omega_{J}}\right)_{L^{\prime}}=\sqrt{\pi}\left(\frac{R_{D e}}{\rho_{e}}\right)^{2} .
$$

The limit of validity of this approximation is similarly defined as the point where $\left(1-\left(\omega / \Omega_{J}\right)_{L^{\prime}}\right)=0.9\left(1-\left(\omega / \Omega_{J}\right)\right)$, and is marked by the upward-pointing tick marks in Fig. 2.

The normalised solutions for the current components then follow from Eqs. (3)-(5), giving

$$
\begin{aligned}
\frac{i_{P}}{i_{P D}} & =2\left(\frac{\rho_{i}}{R_{D i}}\right)\left(1-\frac{\omega}{\Omega_{J}}\right), \quad \text { where } \\
i_{P D} & =\left(\frac{\Sigma_{P}^{* 7} \dot{M}}{2 \pi}\right)^{1 / 8}\left(B_{J} R_{J}\right)^{3 / 4} \Omega_{J}, \\
\frac{I_{P}}{I_{P D}} & =4 \pi\left(\frac{\rho_{i}}{R_{D i}}\right)^{2}\left(1-\frac{\omega}{\Omega_{J}}\right), \quad \text { where } \\
I_{P D} & =\left(\frac{\Sigma_{P}^{* 3} \dot{M}}{2 \pi}\right)^{1 / 4}\left(B_{J} R_{J}^{3}\right)^{1 / 2} \Omega_{J},
\end{aligned}
$$

$$
\begin{aligned}
& \frac{i_{\rho}}{i_{\rho_{D}}}=4\left(\frac{R_{D e}}{\rho_{e}}\right)^{2}\left(1-\frac{\omega}{\Omega_{J}}\right), \quad \text { where } \\
& i_{\rho D}=\left(\frac{\Sigma_{P}^{*} \dot{M}}{2 \pi}\right)^{1 / 2} \Omega_{J}, \\
& \frac{I_{\rho}}{I_{\rho D}}=8 \pi\left(\frac{R_{D e}}{\rho_{e}}\right)\left(1-\frac{\omega}{\Omega_{J}}\right), \quad \text { where } \\
& I_{\rho D}=\left(\frac{\Sigma_{P}^{* 3} \dot{M}}{2 \pi}\right)^{1 / 4}\left(B_{J} R_{J}^{3}\right)^{1 / 2} \Omega_{J}, \\
& \frac{\left(j_{\|} / B\right)}{\left(j_{\|} / B\right)_{D}}=2\left[2\left(\frac{\omega}{\Omega_{J}}\right)-\left(1+4\left(\frac{R_{D e}}{\rho_{e}}\right)^{4}\right)\left(1-\frac{\omega}{\Omega_{J}}\right)\right],
\end{aligned}
$$

where

$$
\left(j_{\|} / B\right)_{D}=\Sigma_{P}^{*} \Omega_{J},
$$

and

$\frac{j_{\| i}}{j_{\| i D}}=2\left[2\left(\frac{\omega}{\Omega_{J}}\right)-\left(1+4\left(\frac{\rho_{i}}{R_{D i}}\right)^{8}\right)\left(1-\frac{\omega}{\Omega_{J}}\right)\right]$,

where

$j_{\| i D}=2 \Sigma_{P}^{*} B_{J} \Omega_{J}$.

We note that Eqs. (21b) and (21d) are equivalent to Hill's (2001) Eq. (A13), while Eqs. (21e) and (21f) are the same as Hill's Eq. (A12). These normalised forms are plotted as solid 

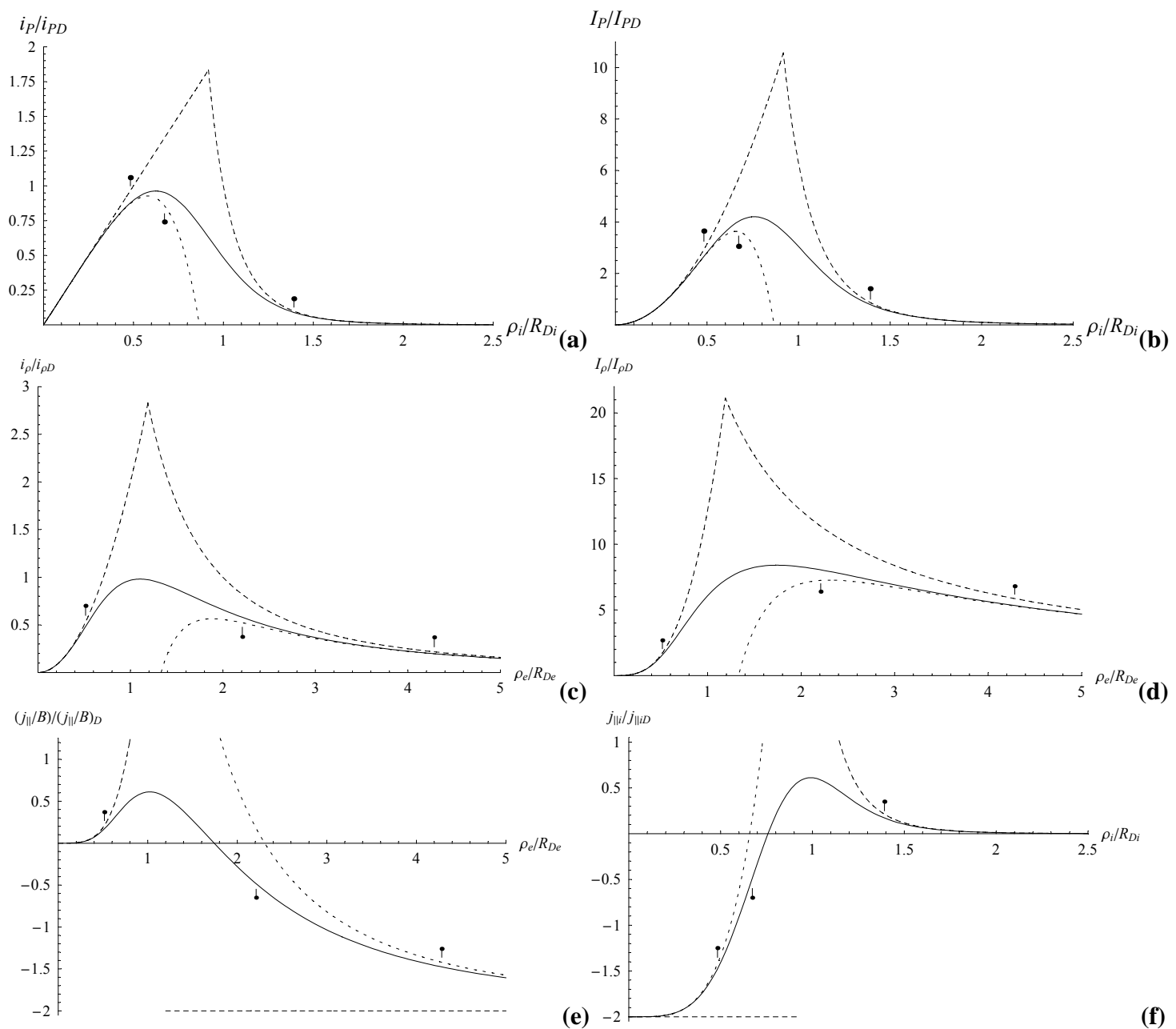

Fig. 3. Plots of normalised steady-state current components for a dipole magnetic field, plotted versus normalised equatorial radial distance $\left(\rho_{e} / R_{D e}\right)$, or normalised distance from the magnetic axis $\left(\rho_{i} / R_{D i}\right)$ in the ionosphere, as appropriate. The plots show (a) the heightintegrated ionospheric Pedersen current intensity, (b) the azimuth- and height-integrated total ionospheric Pedersen current, (c) the current sheet-integrated equatorial radial current intensity, (d) the azimuth- and current sheet-integrated total equatorial radial current, (e) the equatorial field-aligned current density per unit magnetic field strength, and (f) the field-aligned current density just above the ionosphere. The corresponding normalization constants are given by Eq. (21). The solid lines show the full solutions given by Eqs. (14) and (21), while the long-dashed lines show the small and large $\rho_{e}$ forms (the ' $S$ ' and ' $L$ ' approximations), shown to their point of intersection at $\left(\rho_{e} / R_{D e}\right)=\sqrt[4]{2}$ (or equivalently $\left.\left(\rho_{i} / R_{D i}\right)=1 / \sqrt[8]{2}\right)$, where $\left(\omega / \Omega_{J}\right)_{S}=\left(\omega / \Omega_{J}\right)_{L}=0$. The downward-pointing tick marks show the limits of validity of these approximate forms, as shown in Fig. 2. The short-dashed lines show the higher-order large $\rho_{e}$ form (the ' $L$ ', approximation) obtained from Eq. (20), whose limit of validity is indicated by the upward-pointing tick mark, as also shown in Fig. 2.

lines in Fig. 3 versus either $\left(\rho_{e} / R_{D e}\right)$ or $\left(\rho_{i} / R_{D i}\right)$ as appropriate. The values and positions of principal features are also tabulated in Table 1 in normalised units, and in Table 2 in physical units. The dashed lines and tick marks in Fig. 3 then show approximate forms in the same format as Fig. 2 for the angular velocity. Specifically, for small $\rho_{e}$ the long-dashed lines show the currents obtained by introducing Eq. (19) (the ' $S$ ' approximation into Eq. (21)). These currents are the same as Eq. (9) for a dipole field, when expressed in normalised form. This ' $S$ ' approximation is drawn to the point where $\left(\omega / \Omega_{J}\right)_{S}$ falls to zero. Beyond this we draw the currents obtained by introducing $\left(\omega / \Omega_{J}\right)_{L}=0$ into Eq. (21) (the ' $L$ ' approximation), which are the same as Eq. (10) for the dipole, when expressed in normalised form. The long-dashed lines thus represent the current profiles that would be driven by an angular velocity profile given by the ' $S$ ' approximation to the point where falls to zero, with zero being taken beyond. The short-dashed lines then show the profiles obtained by introducing Eq. (20) (the ' $L$ '' approximation) into Eq. (21).

The normalised solutions given above show how the form and amplitude of the plasma angular velocity and currents vary with the system parameters for a dipole field. Specifically, Eqs. (13) and (16) show that the solutions scale spatially in the equatorial plane and in the ionosphere as $\rho_{e} \propto\left(\Sigma_{P}^{*} / \dot{M}\right)^{1 / 4}$ and $\rho_{i} \propto\left(\dot{M} / \Sigma_{P}^{*}\right)^{1 / 8}$, respectively, while 
Table 2. Principal features of the plasma angular velocity and coupling current system for a dipole field in physical units

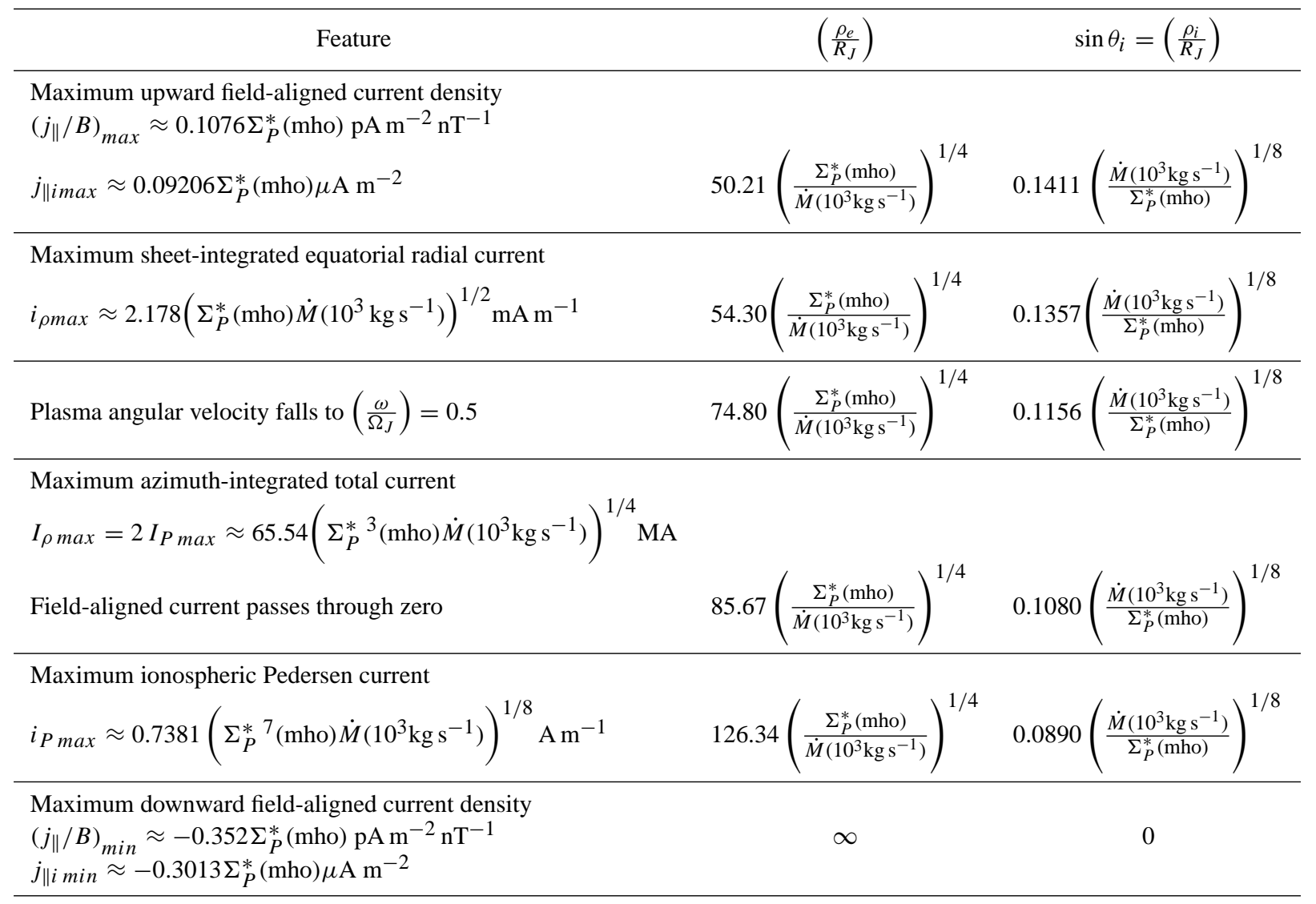

Eq. (21) shows that the amplitude of each component of the current system scales as some power of $\Sigma_{P}^{*}$ and $\dot{M}$ of the form

$i \propto \Sigma_{P}^{*} \frac{(1+\gamma)}{2} \dot{M}^{\frac{(1-\gamma)}{2}}$,

where $\gamma$ is equal to zero for the sheet-integrated equatorial radial current, $1 / 2$ for the azimuth-integrated total fieldperpendicular current, $3 / 4$ for the height-integrated ionospheric Pedersen current, and 1 for the field-aligned current density. The fact that these spatial and amplitude scales combine to produce a linear dependence of the current on $\dot{M}$ for small $\rho_{e}$, as given by Eq. (9), and a linear dependence on $\Sigma_{P}^{*}$ at large $\rho_{e}$, as given by Eq. (10), implies that the currents grow with a specific power of the distance in the inner region, and decline with a specific power of the distance at large distances. It is easy to show that at small distances the currents grow as

$i_{S} \propto \dot{M} \rho_{e}^{2(1+\gamma)} \propto \frac{\dot{M}}{\rho_{i}^{4(1+\gamma)}}$,

while at large distances they decline as

$i_{L} \propto \frac{\Sigma_{P}^{*}}{\rho_{e}^{2(1-\gamma)}} \propto \Sigma_{P}^{*} \rho_{i}^{4(1-\gamma)}$, as may be readily verified by substituting the appropriate form for the angular velocity (i.e. the ' $S$ ' or ' $L$ ' approximations) into Eq. (21). Thus, in summary, the currents grow in the inner region according to Eq. (23a), and depart from this behaviour at an equatorial distance proportional to $\left(\Sigma_{P}^{*} / \dot{M}\right)^{1 / 4}$ (as shown by the 'inner' downward tick marks in Fig. 3), where the current value depends on $\Sigma_{P}^{*}$ and $\dot{M}$ according to Eq. (22). Similarly, the currents decline in the outer region according to Eq. (23b), starting at an equatorial distance proportional to $\left(\Sigma_{P}^{*} / \dot{M}\right)^{1 / 4}$ (as shown by the 'outer' downward tick marks in Fig. 3), where the current value again depends on $\Sigma_{P}^{*}$ and $\dot{M}$ according to Eq. (22).

\section{Plasma angular velocity and coupling current system for a current sheet magnetic model}

The solution for the coupling currents for a dipole field represents an important paradigm case. Nevertheless, the model is unrealistic in its application to Jupiter because the middle magnetosphere field lines are not quasi-dipolar, but are significantly distorted outward from the planet by azimuthal currents flowing in the equatorial plasma, as shown in Fig. 1. Thus field lines at a given radial distance in the equatorial plane map to a significantly lower latitude in the ionosphere 

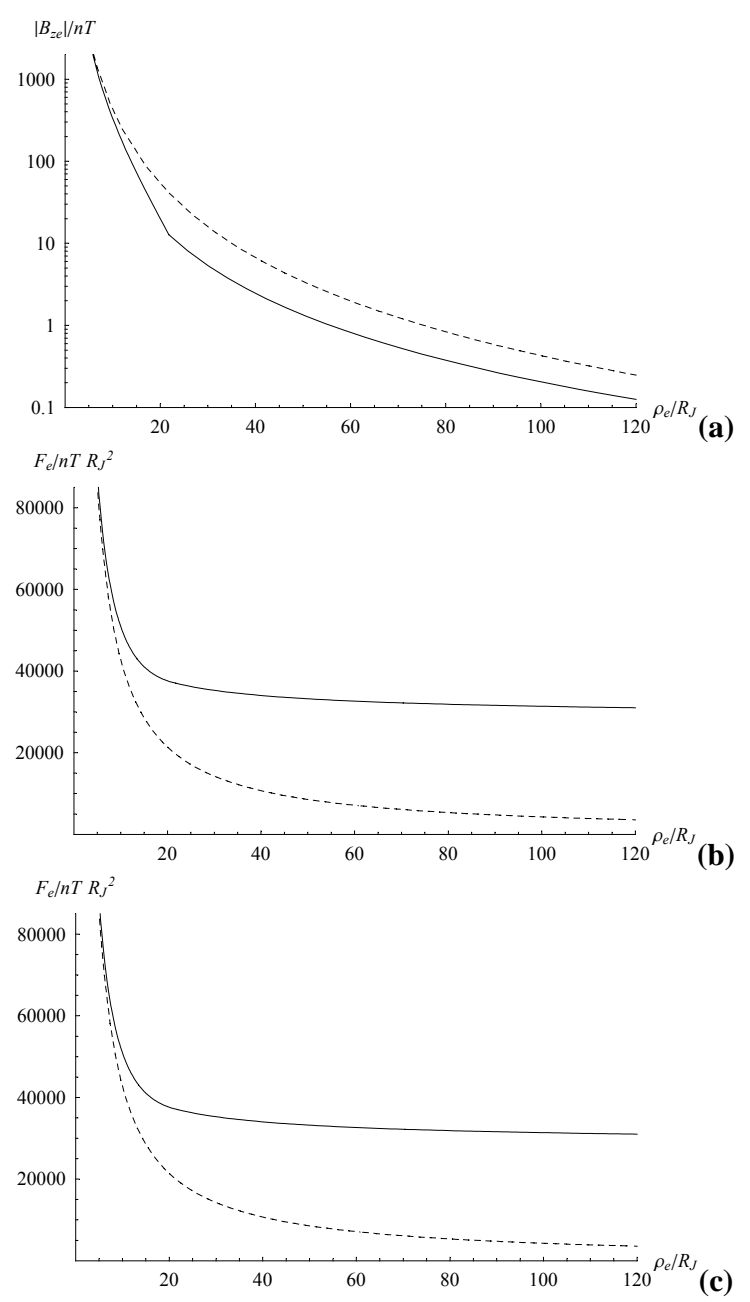

Fig. 4. Plots showing parameters of the current sheet field model employed in Sect. 4 (solid lines), compared with values for the planetary dipole field alone (dashed lines). Plot (a) is a log-linear plot of the modulus of the north-south equatorial magnetic field $\left|B_{z e}\right|$ threading the equatorial plane, shown versus jovicentric equatorial radial distance $\rho_{e}$. This field component is actually negative (i.e. points south) in both cases. The kink in the curve at $\sim 21.78 \mathrm{R}_{\mathrm{J}}$ in the current sheet model marks the point where we switch from the 'CAN' model to the 'KK' model, as discussed in the text. Plot (b) similarly shows the equatorial flux function $F_{e}$ of the model fields versus jovicentric equatorial radial distance $\rho_{e}$. Plot (c) shows the mapping of the field lines between the equatorial plane and the ionosphere, determined from Eq. (1). The ionospheric dipole co-latitude of the field line is plotted versus jovicentric equatorial radial distance $\rho_{e}$.

than for a dipole, thereby increasing the electric field and current for a given departure of the plasma from rigid corotation. In their previous investigations, Cowley and Bunce (2001) and Cowley et al. (2002) employed an empirical model of the equatorial field based on Voyager magnetic data. This model will also be used here, its properties being illustrated in Fig. 4. The solid line in Fig. 4a shows a log-linear plot of the modulus of the equatorial north-south magnetic field
$B_{z e}$ versus equatorial radial distance (the actual values are, of course, all negative), while the dashed line shows the dipole field for comparison. The model field departs from the dipole in the inner part of the middle magnetosphere, and remains significantly lower in magnitude throughout the region of interest, reflecting the outward distension of the current sheet field lines. The model employs the "Voyager-1/Pioneer-10 model" of Connerney et al. (1981) (the "CAN" model) out to a certain radial distance (using the analytic approximations derived by Edwards et al., 2001), and the empirical Voyager-1 outbound model of Khurana and Kivelson (1993) (the "KK" model) beyond. The radial distance at which these models are joined, $\rho_{e}^{*} \sim 21.78 \mathrm{R}_{\mathrm{J}}$, indicated by the "kink" in the solid curve in Fig. 4a, is determined from the intersection of the two model curves, such that there is no discontinuity in the field magnitude at this point (only in the first derivative). The expression for the field in the "CAN" region has been given previously by Cowley and Bunce (2001) and Cowley et al. (2002), and will not be repeated here. The expression for the field in the "KK" region, however, is simply a power law given by

$B_{z e}=-B_{0}\left(\frac{R_{J}}{\rho_{e}}\right)^{m}$,

where $B_{0}=5.4 \times 10^{4} \mathrm{nT}$ and $m=2.71$. This function was determined from a fit to outbound Voyager- 1 data over the range from $\sim 20$ to $\sim 100 \mathrm{R}_{\mathrm{J}}$, corresponding approximately to the range over which we employ it here.

The equatorial flux function $F_{e}$ satisfying Eq. (2) is shown by the solid line in Fig. 4b, where the dashed line again shows the dipole value (Eq. 11b). The value of $F_{e}$ at $\rho_{e}^{*}$ is set by the CAN model, equal to $\sim 3.70 \times 10^{4} \mathrm{nTR}_{\mathrm{J}}^{2}$, while beyond this, in the "KK" region, integration of Eq. (2) using Eq. (24) for $B_{z e}$ yields

$F_{e}\left(\rho_{e}\right)=F_{\infty}+\frac{B_{0} R_{J}^{2}}{(m-2)}\left(\frac{R_{J}}{\rho_{e}}\right)^{m-2}$,

where $F_{\infty}$, the model value of $F_{e}$ at infinity, is $\sim 2.85 \times 10^{4} \mathrm{nTR}_{J}^{2}$. It can be seen in Fig. $4 \mathrm{~b}$ that the value of $F_{e}$ for the current sheet model is much larger than for the dipole at a given equatorial distance, and varies over only a narrow range in the outer middle magnetosphere. Since the value of $F_{e}$ is directly related to the magnetic co-latitude where the field lines map to the ionosphere through Eq. (1), the implication is that the current sheet field lines map to significantly larger co-latitudes than for the dipole, and also to a very narrow co-latitude range. This is shown explicitly in Fig. 4c, where we plot the co-latitude of the field lines in the ionosphere versus equatorial radial distance. In the current sheet model (solid line), the ionospheric mapping varies from a co-latitude of $\sim 16.7^{\circ}$ at $30 \mathrm{R}_{\mathrm{J}}$ to $\sim 15.6^{\circ}$ at $120 \mathrm{R}_{\mathrm{J}}$, a range of only $\sim 1.1^{\circ}$. Even the current sheet field line from infinity, should the model (unrealistically) be taken to extend that far, only maps to $\sim 15.0^{\circ}$. Field lines at smaller co-latitudes then 
do not thread the current sheet in this model, but must map instead to the outer magnetosphere and magnetic tail, regions which are not described by the present theory. In the dipole model, by contrast, equatorial field lines in the range 30 to $120 \mathrm{R}_{\mathrm{J}}$ map between $\sim 10.5^{\circ}$ and $\sim 5.2^{\circ}$, a range of $\sim 5.3^{\circ}$, and go to the pole, of course, at infinity.

Solutions for such a "current sheet" field must generally be computed numerically for specific values of the system parameters, and results have been presented to date by Cowley et al. $(2002,2003)$ for a few spot values. Here we present an approximate analytic solution which applies to the region beyond $\rho_{e}^{*} \sim 21.78 \mathrm{R}_{\mathrm{J}}$, where the field varies with distance as a power law, which previous work has shown to be the main current-carrying region. However, we must first enquire how solutions in the power law region depend on conditions inside the region, where the dipole field is dominant and the transition to the power law takes place. We commented previously for the dipole problem that solutions of the HillPontius equation which are started at an arbitrary angular velocity well within the "Hill distance" converge rapidly onto the solution which rigidly corotates at small distances, such that the behaviour at larger distances is very insensitive to the choice of initial condition. Numerical investigation shows that the solutions for the current sheet field exhibit the same property (Cowley and Bunce, 2002). The implication for the present problem is that, provided the effective "Hill distance" is larger than $\rho_{e}^{*} \sim 20 \mathrm{R}_{\mathrm{J}}$ (i.e. provided the value of $\left(\Sigma_{P}^{*} / \dot{M}\right)$ is not too low), the solutions in the "power law" region will be very insensitive to conditions in the interior region. In this case, we can simply take the power law field to be valid over all distances, but apply the results only to the region outside of $\rho_{e}^{*}$. The validity of this statement may be judged from Fig. 5, where we show solutions for the plasma angular velocity in the inner part of the system spanning $\rho_{e}^{*}$, for three values of $\left(\Sigma_{P}^{*} / \dot{M}\right)$ covering our range of interest, i.e. $10^{-4}, 10^{-3}$ and $10^{-2}$ mho s kg- ${ }^{-1}$. The solid lines show numerical solutions using the full current sheet field model shown in Fig. 4, while the dashed lines similarly show numerical solutions using the power law field over the whole range. (The dot-dashed lines show the analytic approximation to be derived below.) Both numerical solutions were initialised by imposing the near-rigid corotation approximation given by Eq. (8) at $\rho_{e}=5 \mathrm{R}_{\mathrm{J}}$. The position of $\rho_{e}^{*}$ is indicated by the tick mark in each plot, such that both models use the same power law field at larger distances. It can be seen that the two numerical solutions converge rapidly beyond this distance, the convergence becoming increasingly rapid as $\left(\Sigma_{P}^{*} / \dot{M}\right)$ increases. Thus, in the parameter range of interest, the solutions in the power law field region can be approximated by taking the power law field to be valid at all distances. We note that the values of the "Hill distance" corresponding to the values of $\left(\Sigma_{P}^{*} / \dot{M}\right)$ shown in the figure are $27.7,49.2$, and $87.5 \mathrm{R}_{\mathrm{J}}$, thus exceeding $\rho_{e}^{*} \sim 21.78 \mathrm{R}_{\mathrm{J}}$ in each case, though only just so at the lower limit. Convergence of the two solutions is found to break down for lower values of $\left(\Sigma_{P}^{*} / \dot{M}\right) \sim 10^{-5} \mathrm{mhos} \mathrm{kg}^{-1}$, corresponding to a
"Hill distance" of $15.6 \mathrm{R}_{\mathrm{J}}$, at the limit of the parameter range considered here.

We thus consider solutions for the case in which the equatorial field is taken to be given by Eq. (24) at all distances. To obtain an analytic result we also make the further approximation that the flux function is taken to be a constant in the Hill-Pontius equation. Thus, over the region of interest, the equatorial field is taken to map in the ionosphere to a narrow range of distances from the magnetic axis, an approximation shown to be well satisfied for the empirical current sheet model discussed above. While this approximation will generally be valid for "current sheet" fields, it is clearly not valid for quasi-dipolar fields. We thus note that the solutions obtained here do not reduce to the dipole case in the limit that we choose $m=3$ in Eq. (24). With this "current sheet" approximation, then, Eq. (6b) becomes

$\frac{\rho_{e}}{2} \frac{d}{d \rho_{e}}\left(\frac{\omega}{\Omega_{J}}\right)+\left(\frac{\omega}{\Omega_{J}}\right)=2\left(\frac{R_{C S e}}{\rho_{e}}\right)^{m}\left(1-\frac{\omega}{\Omega_{J}}\right)$,

where $R_{C S e}$ is the equatorial "Hill distance" for the power law current sheet field (subscript 'CS')

$\frac{R_{C S e}}{R_{J}}=\left(\frac{2 \pi \Sigma_{P}^{*} B_{0} F_{0}}{\dot{M}}\right)^{1 / m}$.

Here we have put $F_{e}=F_{0}$, a constant, into Eq. (6b), such that the field lines are taken to map in the ionosphere to a fixed distance from the magnetic axis given by $\left(\rho_{i 0} / R_{J}\right)=\sqrt{\left(F_{0} / B_{J} R_{J}^{2}\right)}$ (Eq. 1). The value of $F_{0}$ could be taken for example, to be equal to $F_{\infty}$ in Eq. (25) (in which case $\left(\rho_{i 0} / R_{J}\right)=\left(\rho_{i \infty} / R_{J}\right)$ where $\left(\rho_{i \infty} / R_{J}\right)=$ ${\sqrt{\left(F_{\infty} / B_{J} R_{J}\right)}}^{2} \approx 0.258$, corresponding to a co-latitude of $\sim 14.95^{\circ}$ ), or to some nearby (larger) value representative of the field lines in the region of interest. Equation (26) is then of the same form as Eq. (12) for the dipole field (they are identical when $m=4$ ), from which it is clear that the solutions are functions only of $\rho_{e} / R_{C S e}$, and hence scale with distance as $R_{C S e}$ and with the system parameters as $\left(\Sigma_{P}^{*} / \dot{M}\right)^{1 / m}$. With $m=2.71$, therefore, as used throughout here, the scale length varies somewhat more rapidly with the system parameters than for the dipole, which varies as $\left(\Sigma_{P}^{*} / \dot{M}\right)^{1 / 4}$. The general solution of Eq. (26) can again be found by the integration factor method

$$
\begin{aligned}
& \left(\frac{\omega}{\Omega_{J}}\right)=\left(\frac{4}{m}\right)^{\frac{2}{m}}\left(\frac{R_{C S e}}{\rho_{e}}\right)^{2} \exp \left[\frac{4}{m}\left(\frac{R_{C S e}}{\rho_{e}}\right)^{m}\right] \times \\
& \times\left[\Gamma\left[1-\frac{2}{m}, \frac{4}{m}\left(\frac{R_{C S e}}{\rho_{e}}\right)^{m}\right]+K\right],
\end{aligned}
$$

where $\Gamma(a, z)$ is the incomplete gamma function

$$
\Gamma(a, z)=\int_{z}^{\infty} e^{-t} t^{a-1} d t .
$$

The solutions again diverge at the origin except for the special solution with $K=0$, which rigidly corotates for small 

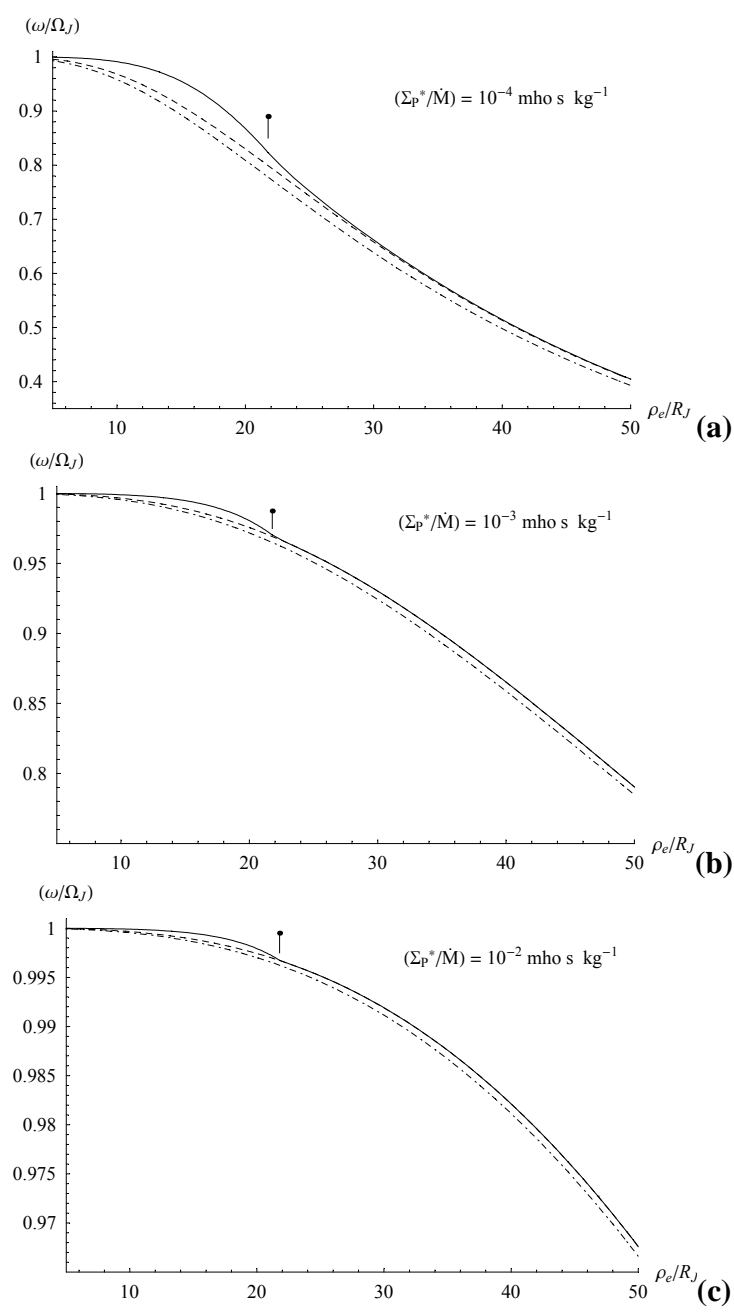

Fig. 5. Plots showing plasma angular velocity profiles versus equatorial radial distance for $\left(\Sigma_{P}^{*} / \dot{M}\right)$ equal to $(\mathbf{a}) 10^{-4}$, (b) $10^{-3}$, and (c) $10^{-2} \mathrm{mho} \mathrm{kg}^{-1}$. The solid lines show the solution obtained by numerical integration of Eq. (6b) using the full 'current sheet' magnetic model shown in Fig. 4, starting from the near-rigid corotation approximation Eq. (8) at $\rho_{e}=5 \mathrm{R}_{\mathrm{J}}$. The tick marks show the point $\left(\rho_{e}^{*} \approx 21.78 \mathrm{R}_{\mathrm{J}}\right)$ where the magnetic field switches from the 'CAN' model to the power-law ' $\mathrm{KK}$ ' model. The dashed lines then show the numerical solution obtained by employing the ' $\mathrm{KK}$ ' power law field (given by Eqs. 24 and 25) over the full distance range, the solutions again being initialised using the appropriate form of Eq. (8) at $\rho_{e}=5 \mathrm{R}_{\mathrm{J}}$. The dot-dashed lines show the approximate analytic solution using the ' $\mathrm{KK}$ ' power law field, given by Eq. (28) with $K=0$ and $m=2.71$. Note that the vertical scale has been tailored to the plot in each case.

$\rho_{e}$. To map the solution to the ionosphere we equate Eqs. (1) and (25), and define an ionospheric scaling distance

$$
\frac{R_{C S i}}{R_{J}}=\frac{1}{2(m-2)}\left(\frac{B_{0}}{B_{J}}\right) \sqrt{\frac{B_{J} R_{J}^{2}}{F_{\infty}}}\left(\frac{\dot{M}}{2 \pi \Sigma_{P}^{*} B_{0} F_{0}}\right)^{1-\frac{2}{m}}
$$

such that the angular velocity mapped to the ionosphere is a function only of

$$
\left(\frac{\Delta \rho_{i}}{R_{C S i}}\right)=\left(\frac{R_{C S e}}{\rho_{e}}\right)^{m-2},
$$

where $\Delta \rho_{i}=\left(\rho_{i}-\rho_{i \infty}\right)$, and $\rho_{i \infty}$ is the distance from the magnetic axis of the field line from infinity as given above. In deriving Eq. (30) we have assumed that $\Delta \rho_{i}$ is small compared with $\rho_{i \infty}$, in keeping with the "current sheet" approximation introduced above. Numerically, for the above power law field we find

$$
\frac{R_{C S e}}{R_{J}} \approx 56.38\left(\frac{\Sigma_{P}^{*}(\mathrm{mho})}{\dot{M}\left(1000 \mathrm{~kg} \mathrm{~s}^{-1}\right)}\right)^{\frac{1}{2.71}}
$$

and

$$
\frac{R_{C S i}}{R_{J}} \approx 0.0197\left(\frac{\dot{M}\left(1000 \mathrm{~kg} \mathrm{~s}^{-1}\right)}{\Sigma_{P}^{*}(\mathrm{mho})}\right)^{\frac{0.71}{2.71}},
$$

where we have chosen to put $F_{0}=F_{e}\left(70 \mathrm{R}_{J}\right) \approx 3.22 \times 10^{4} \mathrm{nT} R_{J}^{2}$, a representative value in the middle magnetosphere current sheet. Thus, as $\left(\Sigma_{P}^{*} / \dot{M}\right)$ varies over the range of interest from $10^{-4}$ to $10^{-2} \mathrm{mho} \mathrm{s} \mathrm{kg}^{-1}$, we find that $R_{C S e}$ varies between 24.1 and $131.9 R_{J}$ (compared with 27.7 to $87.5 R_{J}$ for $R_{D e}$ for the dipole), while $R_{C S i}$ varies between 0.046 and $0.0084 R_{J}$ (compared with 0.19 to $0.11 R_{J}$ for $R_{D i}$ for the dipole). Consequently, since $\left(\rho_{i \infty} / R_{J}\right) \approx 0.258$, as indicated above, we will indeed have $\Delta \rho_{i}$ small compared with $\rho_{i \infty}$ for values $\Delta \rho_{i} \sim R_{C S i}$.

The solid lines in Figs. 6a and b show the normalised angular velocity solution given by Eq. (28) with $K=0$ and $m=2.71$, plotted versus $\left(\rho_{e} / R_{C S e}\right)$ in the equatorial plane, and versus $\left(\Delta \rho_{i} / R_{C S i}\right)$ in the ionosphere, respectively. The form is similar to that for the dipole, though falling away from rigid corotation more quickly (in normalised units) in the inner region, and less quickly in the outer region. This solution is also shown in un-normalised form (with the above value of $F_{0}$ ) by the dot-dashed lines in Fig. 5 , where it is compared with the results of numerical integration of the full solution (solid and dashed lines as described above). It can be seen that the analytic solution forms a very close approximation to the numerical solutions for $\rho_{e}>\rho_{e}^{*}$ under all conditions of interest here, a result we have confirmed by a wider comparative study not illustrated here. The dashed lines and tick marks in Fig. 6 show normalised approximate forms and their regimes of validity, in the same format as Fig. 2. Specifically, the long-dashed lines show the small$\rho_{e}(' S$ ') approximation given by Eq. (8)

$$
\left(\frac{\omega}{\Omega_{J}}\right)_{S}=1-\frac{1}{2}\left(\frac{\rho_{e}}{R_{C S e}}\right)^{m}=1-\frac{1}{2}\left(\frac{R_{C S i}}{\Delta \rho_{i}}\right)^{\frac{m}{m-2}},
$$

where we again note that the series generated by Eq. (7) (of which Eq. (32) is the leading term) is the same as that obtained by asymptotic expansion of the gamma function in Eq. (28) for large argument. The short-dashed lines 


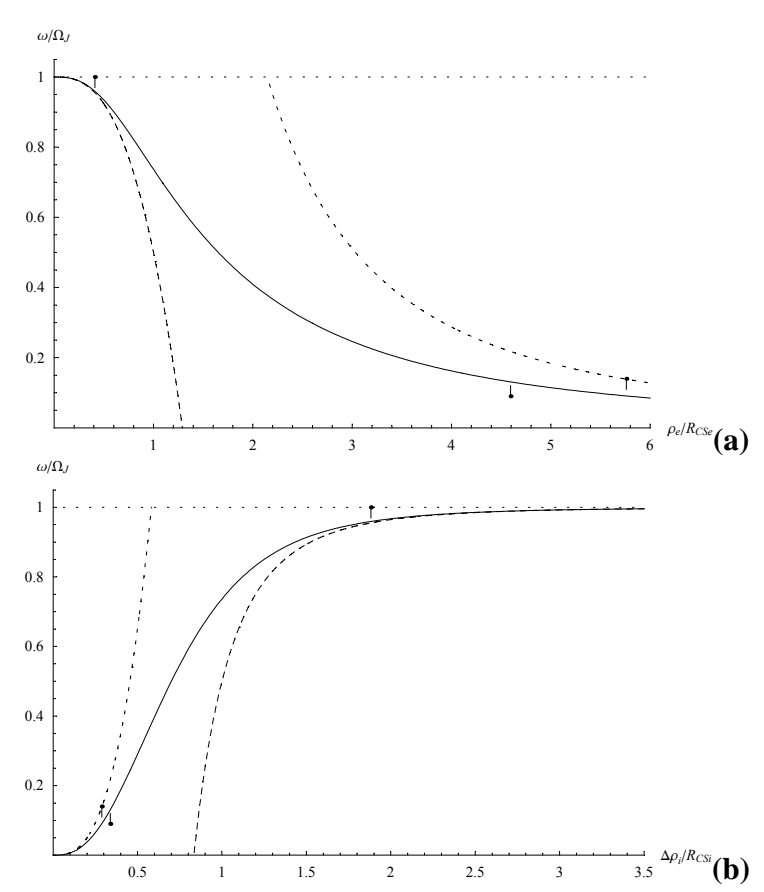

Fig. 6. Plots of the approximate analytic plasma angular velocity profile for the current sheet power law magnetic field model, given by Eq. (24) with $m=2.71$, shown (a) versus $\left(\rho_{e} / R_{C s e}\right)$ in the equatorial plane, and (b) versus $\left(\Delta \rho_{i} / R_{C s i}\right)$ in the ionosphere. The solid line in each case shows the full solution obtained from Eq. (28) with $K=0$, such that the plasma rigidly corotates at small radial distances. The long-dashed lines show the small- $\rho_{e}$ (' $S$ ') form given by Eq. (32), while the large- $\rho_{e}$ (' $L$ ') form is just $\left(\omega / \Omega_{J}\right)_{L}=0$. The downward-pointing tick marks indicate the limits of validity of both these approximations as defined for the dipole case shown in Fig. 2. The short-dashed lines show the higher-order large- $\rho_{e}\left({ }^{\prime} L^{\prime \prime}\right.$ ') form given by Eq. (33), whose limit of validity (defined as for the dipole case) is indicated by the upward-pointing tick mark. The horizontal dotted line indicates the condition for rigid corotation, $\left(\omega / \Omega_{J}\right)=1$.

then show the higher-order large- $\rho_{e}\left({ }^{\prime} L^{\prime \prime}\right)$ approximation, obtained from Eq. (28) as

$$
\left(\frac{\omega}{\Omega_{J}}\right)_{L^{\prime}}=\left(\frac{4}{m}\right)^{\frac{2}{m}} \Gamma\left(1-\frac{2}{m}\right)\left(\frac{R_{C S e}}{\rho_{e}}\right)^{2} .
$$

The lower-order large- $\rho_{e}\left({ }^{\prime} L '\right)$ approximation is again simply $\left(\omega / \Omega_{J}\right)_{L}=0$.

The normalised approximate solutions for the currents then follow from Eqs. (3)-(5)

$$
\begin{aligned}
\frac{i_{P}}{i_{P C S}} & =2\left(1-\frac{\omega}{\Omega_{J}}\right), \quad \text { where } \\
i_{P C S} & =\sqrt{\frac{F_{0}}{B_{J} R_{J}^{2}}} \Sigma_{P}^{*} B_{J} R_{J} \Omega_{J}, \\
\frac{I_{P}}{I_{P C S}} & =4 \pi\left(1-\frac{\omega}{\Omega_{J}}\right), \quad \text { where }
\end{aligned}
$$

$$
\begin{aligned}
& I_{P C S}=\left(\frac{F_{0}}{B_{J} R_{J}^{2}}\right) \Sigma_{P}^{*} B_{J} R_{J}^{2} \Omega_{J}, \\
& \frac{i_{\rho}}{i_{\rho C S}}=4\left(\frac{R_{C S e}}{\rho_{e}}\right)\left(1-\frac{\omega}{\Omega_{J}}\right), \quad \text { where } \\
& i_{\rho C S}=\left(\frac{F_{0}}{B_{J} R_{J}^{2}}\right)\left(\frac{\dot{M}}{2 \pi \Sigma_{P}^{*} B_{0} F_{0}}\right)^{1 / m} \Sigma_{P}^{*} B_{J} R_{J} \Omega_{J}, \\
& \frac{I_{\rho}}{I_{\rho C S}}=8 \pi\left(1-\frac{\omega}{\Omega_{J}}\right), \quad \text { where } \\
& I_{\rho C S}=\left(\frac{F_{0}}{B_{J} R_{J}^{2}}\right) \Sigma_{P}^{*} B_{J} R_{J}^{2} \Omega_{J}, \\
& \frac{\left(j_{\|} / B\right)}{\left(j_{\|} / B\right)_{C S}}=4\left(\frac{\rho_{e}}{R_{C S e}}\right)^{m-2} \times \\
& \times\left[\left(\frac{\omega}{\Omega_{J}}\right)-2\left(\frac{R_{C s e}}{\rho_{e}}\right)^{m}\left(1-\frac{\omega}{\Omega_{J}}\right)\right], \quad \text { where } \\
& \left(j_{\|} / B\right)_{C S}=\left(\frac{B_{J}}{B_{0}}\right)\left(\frac{F_{0}}{B_{J} R_{J}^{2}}\right) \times \\
& \times\left(\frac{2 \pi \Sigma_{P}^{*} B_{0} F_{0}}{\dot{M}}\right)^{1-\frac{2}{m}} \Sigma_{P}^{*} \Omega_{J}, \quad \text { and } \\
& \frac{j_{\| i}}{j_{\| i C S}}=4\left(\frac{R_{C S i}}{\Delta \rho_{i}}\right) \times \\
& \times\left[\left(\frac{\omega}{\Omega_{J}}\right)-2\left(\frac{\Delta \rho_{i}}{R_{C S i}}\right)^{\frac{m}{m-2}}\left(1-\frac{\omega}{\Omega_{J}}\right)\right], \quad \text { where } \\
& j_{\| i C S}=2\left(\frac{B_{J}}{B_{0}}\right)\left(\frac{F_{0}}{B_{J} R_{J}^{2}}\right) \times \\
& \times\left(\frac{2 \pi \Sigma_{P}^{*} B_{0} F_{0}}{\dot{M}}\right)^{1-\frac{2}{m}} \Sigma_{P}^{*} B_{J} \Omega_{J} .
\end{aligned}
$$

These forms are shown by the solid lines in Fig. 7, plotted versus either $\left(\rho_{e} / R_{C s i}\right)$ or $\left(\rho_{i} / R_{C s i}\right)$ as appropriate. The dashed lines show approximate forms based on the ' $S$ ', ' $L$ ', and ' $L$ ', approximations for the angular velocity, in the same format as Fig. 3 for the dipole. Comparison with Fig. 3 shows similarities, but also major differences with the currents for the dipole field. The differences arise from the fact that the current sheet field lines reach the ionosphere in a narrow band at a finite co-latitude, rather than continuously approaching the pole with increasing radial distance, as for the dipole. The ionospheric Pedersen current (Eq. 34a), while being proportional to the displacement of the band from the magnetic axis $\sqrt{F_{0} / B_{J} R_{J}^{2}}$, then varies with co-latitude only through the variation of the plasma angular velocity. As seen in Fig. 7a, the Pedersen current, therefore, peaks at the poleward edge of the band, where the angular velocity is zero, 

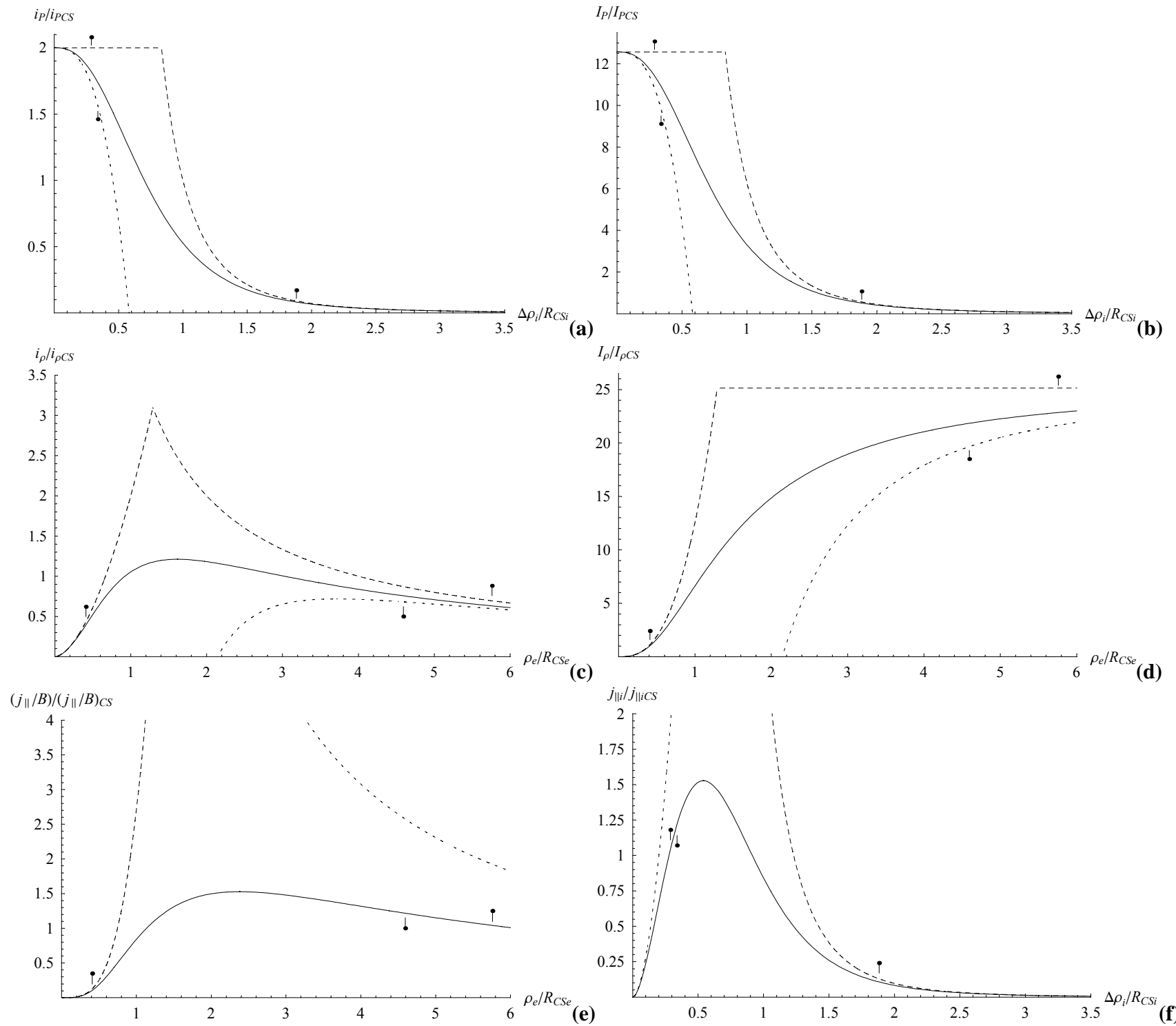

Fig. 7. Plots of normalised steady-state current components for a power law equatorial magnetic field (Eq. (24) with $m=2.71)$ and 'current sheet' approximate mapping to the ionosphere, plotted versus $\left(\rho_{e} / R_{C S e}\right)$ or $\left(\Delta \rho_{i} / R_{C S i}\right)$ as appropriate. As in Fig. 3 for the dipole, the plots show (a) the height-integrated ionospheric Pedersen current intensity, (b) the azimuth- and height-integrated total ionospheric Pedersen current, (c) the current sheet-integrated equatorial radial current intensity, (d) the azimuth- and current sheet-integrated total equatorial radial current, (e) the equatorial field- aligned current density per unit magnetic field strength, and (f) the field-aligned current density just above the ionosphere. The normalization constants are given by Eq. (34). The solid lines show the full approximate solutions given by Eqs. (28) and (34), while the long-dashed lines show the small and large- $\rho_{e}$ forms derived from $\left(\omega / \Omega_{J}\right)_{S}$ given by Eq. $(32)$ and $\left(\omega / \Omega_{J}\right)_{L}=0$, drawn to their point of intersection at $\left(\rho_{e} / R_{C S e}\right)=\sqrt[m]{2}$. The downward-pointing tic-marks show the limits of validity of these approximate forms as defined for the dipole case. The short-dashed lines show the higher-order large- $\rho_{e}$ form given by Eq. (33), whose limit of validity is indicated by the upward-pointing tick mark, again defined as for the dipole case.

and falls monotonically with distance from the boundary as the angular velocity approaches rigid corotation (Fig. 6b). This implies that the azimuth-integrated total current also varies monotonically with distance, the total equatorial current (Eq. 34d) thus rising with increasing equatorial distance towards $8 \pi F_{0} \Sigma_{P}^{*} \Omega_{J}$ (strictly, $8 \pi F_{\infty} \Sigma_{P}^{*} \Omega_{J}$ ) at infinity, as seen in Fig. 7d. This behaviour also implies that the radial current intensity (Eq. 34c) rises to a peak value with increas- ing distance, and then falls as $\rho_{e}^{-1}$ at large distances, as seen in Fig. 7c. The further implication of a monotonically increasing total current is that the field-aligned current is unidirectional, flowing consistently from the ionosphere to the equatorial current sheet, as shown in Figs. 7e and f. Closure of the current system must then occur outside the region described by the model, on field lines mapping between the ionosphere at higher latitudes and the outer magnetosphere 
Table 3. Principal features of the plasma angular velocity and coupling current system for the power law current sheet field in normalised units, obtained (with $m=2.71$ ) from the approximate analytic solution of Sect. 4

\begin{tabular}{lccc}
\hline \multicolumn{1}{c}{ Feature } & $\left(\frac{\rho_{e}}{R_{C s e}}\right)$ & $\left(\frac{\Delta \rho_{i}}{R_{C s i}}\right)$ & $\left(\frac{\omega}{\Omega_{J}}\right)$ \\
\hline $\begin{array}{l}\text { Maximum sheet-integrated equatorial radial current } \\
\left(i_{\rho} / i_{\rho C S}\right)_{\text {max }} \approx 1.2109\end{array}$ & 1.6142 & 0.7118 & 0.5113 \\
\hline Plasma angular velocity falls to $\left(\frac{\omega}{\Omega_{J}}\right)=0.5$ & 1.6521 & 0.7002 & 0.5 \\
\hline $\begin{array}{l}\text { Maximum upward field-aligned current density } \\
\left(\left(j_{\|} / B\right) /\left(j_{\|} / B\right)_{C S}\right)_{\text {max }}=\left(j_{\| i} / j_{\| i C S}\right)_{\text {max }} \approx 1.5274\end{array}$ & 2.3777 & 0.5407 & 0.3339 \\
\hline $\begin{array}{l}\text { Maximum height-integrated ionospheric Pedersen } \\
\text { current }\left(i_{P} / i_{P C S}\right)_{\text {max }}=2\end{array}$ & & & \\
$\begin{array}{l}\text { Maximum azimuth-integrated total current } \\
\left(I_{\rho} / I_{\rho C S}\right)_{\text {max }}=2\left(I_{P} / I_{P C S}\right)_{\text {max }}=8 \pi \approx 25.133\end{array}$ & $\infty$ & 0 & 0 \\
\begin{tabular}{l} 
Field-aligned current goes to zero \\
\hline
\end{tabular} & & & \\
\hline
\end{tabular}

Table 4. Principal features of the plasma angular velocity and coupling current system for the power law current sheet field in physical units, obtained (using $B_{0}=5.4 \times 10^{4} \mathrm{nT}, m=2.71, F_{\infty} \approx 2.85 \times 10^{4} \mathrm{nT} \mathrm{R}_{\mathrm{j}}^{2}$ and $F_{0} \approx 3.22 \times 10^{4} \mathrm{nT} \mathrm{R} \mathrm{j}_{\mathrm{j}}^{2}$ ) from the approximate analytic solution of Sect. 4

\begin{tabular}{|c|c|c|}
\hline Feature & $\left(\frac{\rho_{e}}{R_{J}}\right)$ & $\left(\frac{\Delta \rho_{i}}{R_{J}}\right)$ \\
\hline $\begin{array}{l}\text { Maximum sheet-integrated equatorial radial current } \\
i_{\rho \max } \approx 8.690\left(\Sigma_{P}^{*}(\mathrm{mho})^{1.71} \dot{\mathrm{M}}\left(10^{3} \mathrm{~kg} \mathrm{~s}^{-1}\right)\right)^{\frac{1}{2.71}} \mathrm{~mA} \mathrm{~m}^{-1}\end{array}$ & $91.02\left(\frac{\Sigma_{P}^{*}(\mathrm{mho})}{\dot{M}\left(10^{3} \mathrm{~kg} \mathrm{~s}^{-1}\right)}\right)^{1 / 2.71}$ & $0.01400\left(\frac{\dot{M}\left(10^{3} \mathrm{~kg} \mathrm{~s}^{-1}\right)}{\Sigma_{P}^{*}(\mathrm{mho})}\right)^{\frac{0.71}{2.71}}$ \\
\hline Plasma angular velocity falls to $\left(\frac{\omega}{\Omega_{J}}\right)=0.5$ & $93.15\left(\frac{\Sigma_{P}^{*}\left(\mathrm{mho}^{2}\right)}{\dot{M}\left(10^{3} \mathrm{~kg} \mathrm{~s}^{-1}\right)}\right)^{1 / 2.71}$ & $0.01377\left(\frac{\dot{M}\left(10^{3} \mathrm{~kg} \mathrm{~s}^{-1}\right)}{\Sigma_{P}^{*}(\mathrm{mho})}\right)^{\frac{0.71}{2.71}}$ \\
\hline $\begin{array}{l}\text { Maximum upward field-aligned current density } \\
\left(j_{\|} / B\right)_{\text {max }} \approx \\
2.808\left(\Sigma_{P}^{*}(\mathrm{mho})^{3.42} \dot{M}\left(10^{3} \mathrm{~kg} \mathrm{~s}^{-1}\right)^{-0.71}\right)^{\frac{1}{2.71}} \mathrm{pA} \mathrm{m}^{-2} \mathrm{nT}^{-1} \\
j_{\| \text {i } \max } \approx 2.404\left(\Sigma_{P}^{*}(\mathrm{mho})^{3.42} \dot{M}\left(10^{3} \mathrm{~kg} \mathrm{~s}^{-1}\right)^{-0.71} \mu \mathrm{Am}^{-2}\right.\end{array}$ & $134.06\left(\frac{\Sigma_{P}^{*}\left(\mathrm{mho}^{3}\right)}{\dot{M}\left(10^{3} \mathrm{~kg} \mathrm{~s}^{-1}\right)}\right)^{1 / 4}$ & $0.01063\left(\frac{\dot{M}\left(10^{3} \mathrm{~kg} \mathrm{~s}^{-1}\right)}{\Sigma_{P}^{*}(\mathrm{mho})}\right)^{\frac{0.71}{2.71}}$ \\
\hline $\begin{array}{l}\text { Maximum height-integrated ionospheric Pedersen current } \\
i_{P \max } \approx 2.950 \Sigma_{P}^{*}(\mathrm{mho}) \mathrm{A} \mathrm{m}^{-1} \\
\text { Maximum azimuth-integrated total current } \\
I_{\rho \max }=2 I_{P \max } \approx 725.8 \Sigma_{P}^{*}(\text { mho }) \mathrm{MA} \\
\text { Field-aligned current goes to zero }\end{array}$ & $\infty$ & 0 \\
\hline
\end{tabular}

and magnetic tail. The values and positions of principal features of the solution are again tabulated in Table 3 in normalised units, and in Table 4 in physical units.

The behaviour of these approximate solutions thus reflects the results presented previously by Cowley and Bunce (2001) and Cowley et al. (2002, 2003), using the full current sheet field model shown in Fig. 4. One minor difference is that in the numerical solutions the total equatorial (and ionospheric) current rises with increasing distance to a maximum value slightly above $8 \pi F_{\infty} \Sigma_{P}^{*} \Omega_{J}$ before falling with decreasing $F$ to the latter value at infinity, rather than following the strictly monotonically rising behaviour of the approximation. Correspondingly, the field-aligned current in the numerical solutions reverses to small negative values (given by Eqs. 10e and $\mathrm{f}$ ) at large radial distances (and hence, close to the poleward boundary in the ionosphere), rather than going to zero as in the approximation (Fig. 7f), though the net current closure is small. However, for the range of system parameters considered here, the maximum in the total current and the concurrent reversal of the field-aligned current typically take 
place at equatorial distances of several hundred to several thousand $\mathrm{R}_{\mathrm{J}}$, far beyond the limit of physical applicability of the model. Within the region of applicability, the agreement between the numerical and approximate analytic results is found to be very good.

In like manner to the dipole results, the normalised solutions given above show how the form and amplitude of the plasma angular velocity and currents vary with the system parameters in the case of a power law current sheet field. Equations (27) and (29) show that the solutions scale spatially in the equatorial plane and in the ionosphere as $\rho_{e} \propto\left(\Sigma_{P}^{*} / \dot{M}\right)^{1 / m}$ and $\Delta \rho_{i} \propto\left(\Sigma_{P}^{*} / \dot{M}\right)^{1-2 / m}$, respectively, while Eq. (34) shows that the amplitude of each component of the current system scales as some power of $\Sigma_{P}^{*}$ and $\dot{M}$ of the same form as Eq. (22), but with $\gamma$ equal to $1-2 / m$ for the equatorial radial current, 1 for the Pedersen current and azimuth-integrated total field-perpendicular current (such that these currents scale linearly with $\Sigma_{P}^{*}$ and are independent of $\dot{M}$ ), and 3-4/m for the field-aligned current density. Since these values of $\gamma$ are consistently higher for a given current component for the current sheet model than for the dipole (at least for $m>2$, as investigated here), the implication is that the currents scale as a somewhat higher power of the conductivity for the current sheet model than for the dipole, and as a somewhat lower power of the mass outflow rate. The corresponding behaviours at small and large distances, such that the solutions obey Eqs. (9) and (10), are

$i_{S} \propto \dot{M} \rho_{e}^{\frac{m}{2}(1+\gamma)} \propto \frac{\dot{M}}{\Delta \rho_{i}^{(m(1+\gamma) / 2(m-2))}}$

and

$i_{L} \propto \frac{\Sigma_{P}^{*}}{\rho_{e}^{m(1-\gamma) / 2}} \propto \Sigma_{P}^{*} \Delta \rho_{i}^{(m(1-\gamma) / 2(m-2))}$,

as can be verified by substituting the appropriate approximations (' $S$ ' and ' $L$ ') for the angular velocity into Eq. (34). Thus, for example, with $\gamma=1-2 / m$, the equatorial radial current increases as $\rho_{e}^{m-1}$ in the inner region and falls as $\rho_{e}^{-1}$ at large distances, while the field-aligned current with $\gamma=3-4 / m$ grows as $\rho_{e}^{2(m-1)}$ in the equatorial plane in the inner region and approaches zero in this approximation at large distances, as indicated above (Figs. 7e, f). The spatial variation of the field-aligned current in the large-distance limit may then be obtained from the higher-order large-distance approximation (Eq. 33), from which it is found that the current varies as $\rho_{e}^{-(4-m)}$ in the equatorial plane, and as $\Delta \rho_{i}^{(4-m) /(m-2)}$ in the ionosphere.

\section{Comparison of system behaviour for the dipole and current sheet field models}

In this section we finally provide a summary and comparison of how the major features of the plasma flow and coupling current system vary with $\Sigma_{P}^{*}$ and $\dot{M}$ for the dipole and current sheet field models. Specifically, we consider the location of corotation breakdown, the magnitudes and locations of the peak values of the various current components, and the latitudinal width in the ionosphere of the region of upwarddirected field-aligned current. With the exception of the latter parameter, in effect we here provide plots showing how the quantities in Tables 2 (for the dipole) and 4 (for the power law field approximation) vary with $\Sigma_{P}^{*}$ and $\dot{M}$. We also compare the approximate results for the current sheet field with spot values obtained by numerical integration using the full current sheet field.

Figure 8 shows how the spatial scale on which plasma corotation breaks down depends on $\Sigma_{P}^{*}$ and $\dot{M}$ for the two models. Specifically, we show the position where $\left(\omega / \Omega_{J}\right)=$ 0.5 , as previously given in Tables $1-4$. In Fig. 8a the equatorial distance is plotted versus $\Sigma_{P}^{*}$ in $\log$-log format for $\dot{M}=100,1000$ and $10000 \mathrm{~kg} \mathrm{~s}^{-1}$, while in Fig. $8 \mathrm{~b}$ it is plotted versus $\dot{M}$ in similar format for $\Sigma_{P}^{*}=0.1,1$ and 10 mho. Solid lines give results for the dipole field obtained from Eqs. (13) and (14), showing that the distance increases with the conductivity as $\Sigma_{P}^{* 1 / 4}$, and decreases with the mass outflow rate as $\dot{M}^{-1 / 4}$. The dashed lines show corresponding results obtained from the power law field approximate solutions Eqs. (27) and (28) (with $m=2.71$ and $F_{0} \approx 3.22 \times 10^{4} \mathrm{nTR} \mathrm{R}_{\mathrm{J}}^{2}$ as above), which, of course, are not applicable to the full field model at distances smaller than $\rho_{e}^{*} \approx 21.78 \mathrm{R}_{\mathrm{J}}$. These increase more rapidly with $\Sigma_{P}^{*}$ and decrease more rapidly with $\dot{M}$, as $\Sigma_{P}^{* 1 / 2.71}$ and $\dot{M}^{-1 / 2.71}$, respectively. Overall, however, the equatorial distances of corotation breakdown are similar for the dipole and current sheet fields as noted above, but are generally somewhat larger for the current sheet model than for the dipole, particularly for larger values of $\Sigma_{P}^{*}$ and smaller values of $\dot{M}$. The solid dots in the figures provide spot values obtained by numerical integration of the full current sheet solution, their close association with the dashed lines clearly indicating the values of $\dot{M}$ (in Fig. 8a) and $\Sigma_{P}^{*}$ (in Fig. 8b) employed. This close association also confirms that the analytic solutions provide good approximations to the numerical results in the power law regime over essentially the whole parameter range considered here. The only notable deviations occur at small $\Sigma_{P}^{*}$ and large $\dot{M}$, where corotation breakdown occurs at equatorial distances approaching the radial limit of the power law field region at $\rho_{e}^{*} \approx 21.78 \mathrm{R}_{\mathrm{J}}$. In this case the numerical results give somewhat larger distances than the analytic approximation, as also seen in Fig. 5. Corresponding results projected to the ionosphere are shown in Figs. $8 \mathrm{c}$ and $8 \mathrm{~d}$, in a similar format. The horizontal dotted line at $\theta_{i} \approx 14.95^{\circ}$ shows the co-latitude of the current sheet field line from infinity (the corresponding limit for the dipole being, of course, the pole at $\theta_{i}=0^{\circ}$ ). These plots again emphasise the significantly larger distance from the magnetic axis at which plasma corotation breaks down in the ionosphere for the current sheet field than for dipole, despite the similarity of the equatorial results. They also display the relative lack of response of this distance to the system parameters in the current sheet model, this forming the basis of the "current sheet" approximation $F_{e} \approx F_{0}$ employed to obtain the analytic results 


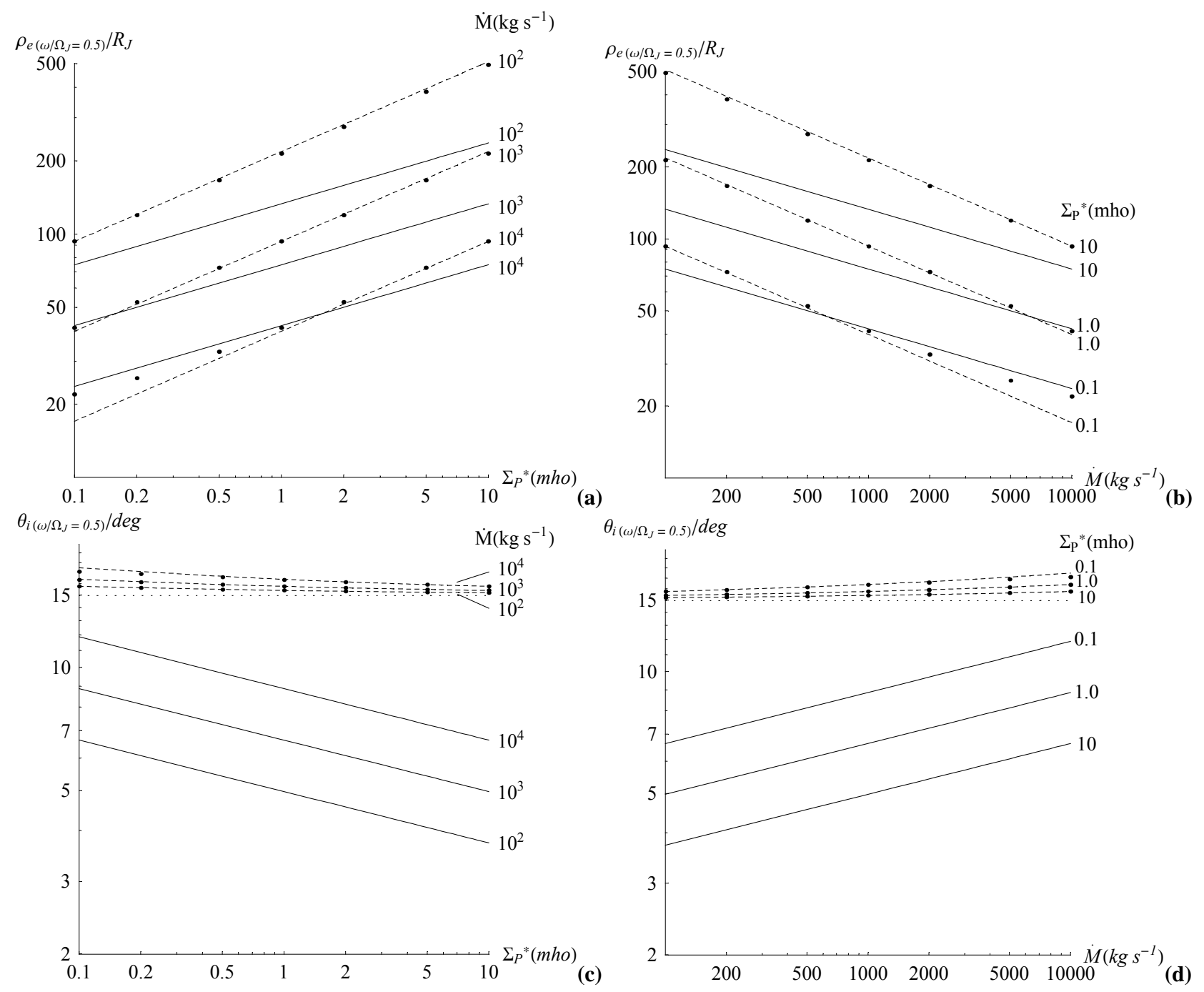

Fig. 8. Plots showing the location of plasma corotation breakdown, specifically where $\left(\omega / \Omega_{J}\right)=0.5$, and its dependence on the system parameters $\Sigma_{P}^{*}$ and $\dot{M}$. Plot (a) shows the equatorial distance at which $\left(\omega / \Omega_{J}\right)=0.5$ versus $\Sigma_{P}^{*}$ in $\log$-log format for $\dot{M}=100,1000$ and $10000 \mathrm{~kg} \mathrm{~s}^{-1}$, while (b) shows this distance similarly plotted versus $\dot{M}$ for $\Sigma_{P}^{*}=0.1,1$ and 10 mho. Solid lines give results for the dipole field obtained from Eqs. (13) and (14), while the dashed lines show corresponding results derived from the power law field approximate solutions for the current sheet model Eqs. (27) and (28). The solid dots provide spot values obtained from numerical integration of the full current sheet solution, whose $\dot{M}$ (in (a)) and $\Sigma_{P}^{*}$ (in (b)) values are obvious from their close association with the corresponding dashed lines. Corresponding plots of the ionospheric co-latitude at which $\left(\omega / \Omega_{J}\right)=0.5$ are shown versus $\Sigma_{P}^{*}$ and $\dot{M}$ in (c) and (d). The horizontal dotted line shows the latitude of the field line from infinity in the current sheet field model.

\section{in Sect. 4.}

Turning now to the current components, in Fig. 9 we show the behaviour of the peak Pedersen current, plotted in a similar format to Fig. 8. The magnitude of the peak current, plotted versus $\Sigma_{P}^{*}$ and $\dot{M}$ in Figs. 9 a and 9 b, respectively, shows that for the dipole (solid lines) the peak Pedersen current increases with the conductivity as $\Sigma_{P}^{* 7 / 8}$, while also increasing weakly with the mass outflow rate as $\dot{M}^{1 / 8}$ (Eq. (21a)), while for the current sheet (dashed line) approximations the peak current varies linearly with $\Sigma_{P}^{*}$ but is independent of $\dot{M}$ (Eq. (34a)). The modestly lower numericallydetermined spot values in the latter case result from our tak- ing $F_{0}=F_{e}\left(\rho_{e}=70 R_{J}\right)$ in the approximation, as above. Very close agreement would have been obtained if we had instead taken $F_{0}=F_{\infty}$. These plots also show that for given system parameters the peak Pedersen current for the current sheet model exceeds that for the dipole by relatively constant factors of $\sim 3$ to $\sim 5$ (typically $\sim 4$ ). This difference arises from the different ionospheric mappings of corotation breakdown, as shown in Figs. 8c and 8d. Figures 9c and 9d show the co-latitude of the peak Pedersen current, which for the dipole field lies typically at $\sim 5^{\circ}$ and is such that the distance from the magnetic axis varies with the system parameters as $\Sigma_{P}^{*-1 / 8}$ and $\dot{M}^{1 / 8}$ (Eq. 16), while for the current sheet ap- 

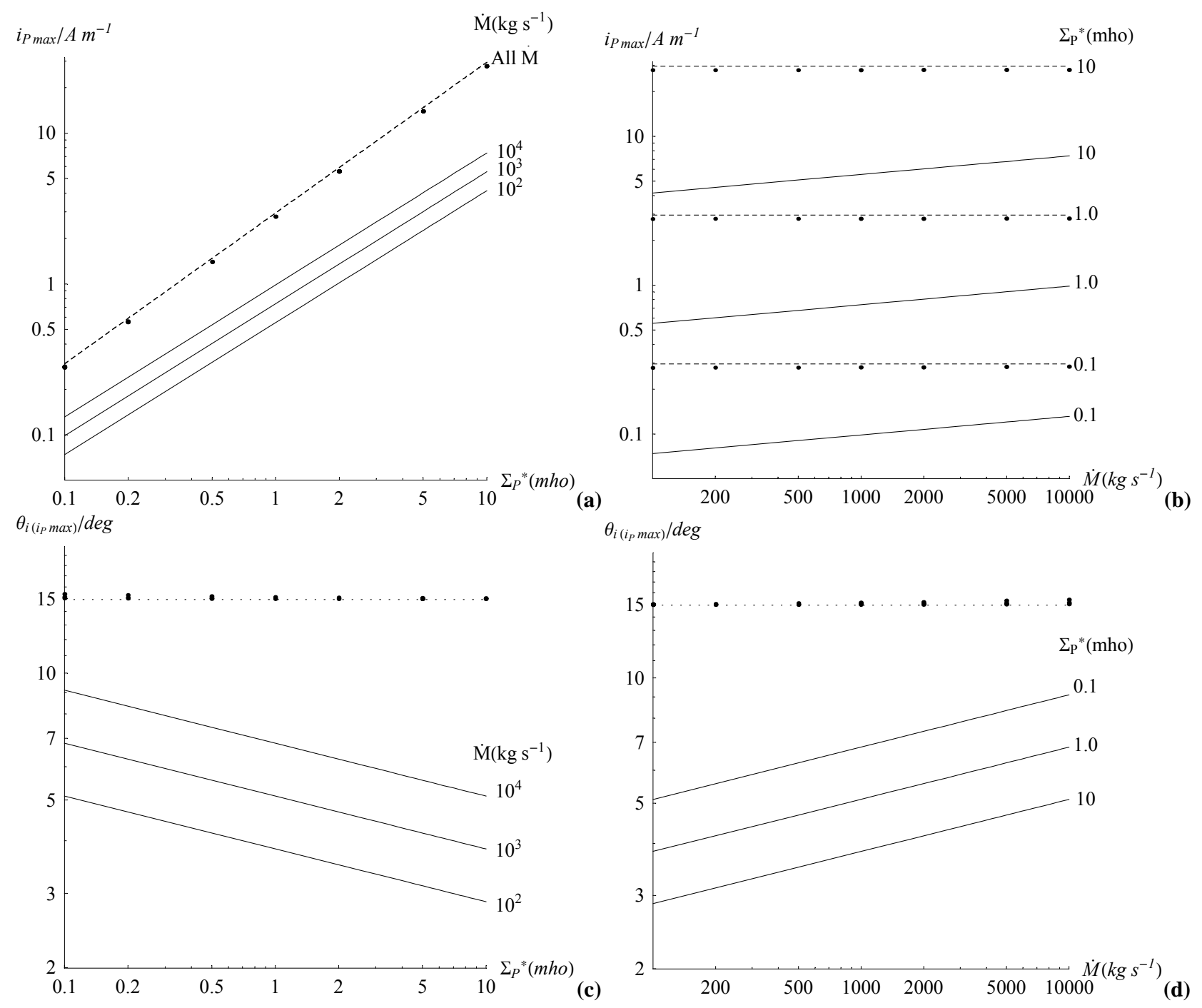

$\theta_{i\left(i_{p} \max \right)} / d e g$

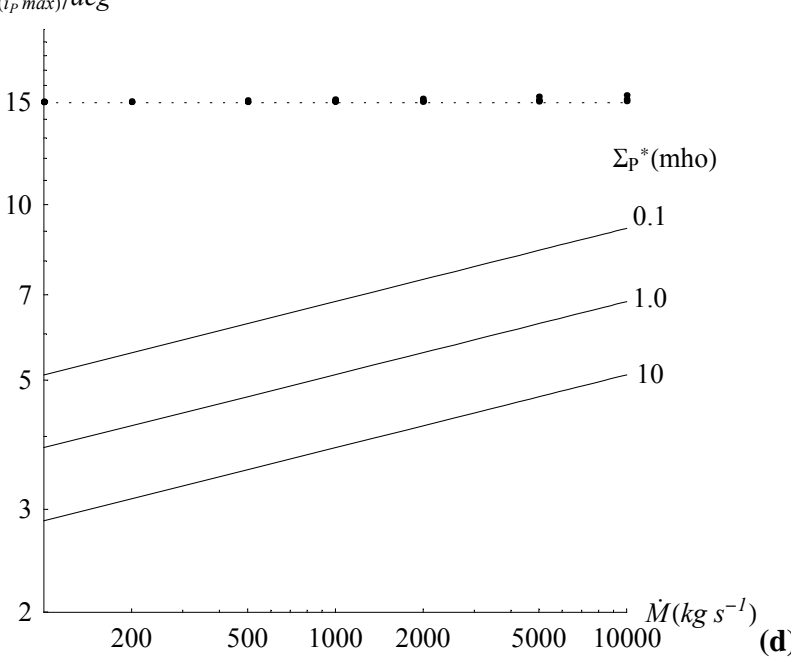

Fig. 9. Plots showing the magnitude and location of the peak height-integrated Pedersen current intensity in the ionosphere, and their dependence on $\Sigma_{P}^{*}$ and $\dot{M}$. Plot (a) shows the magnitude of the peak Pedersen current plotted versus $\Sigma_{P}^{*}$ in log-log format for $\dot{M}=100$, 1000 and $10000 \mathrm{~kg} \mathrm{~s}^{-1}$, while plot (c) show the ionospheric location of the peak. Plots (b) and (d) similarly show the magnitude and location of the peak Pedersen current plotted versus $\dot{M}$ for $\Sigma_{P}^{*}=0.1,1$ and 10 mho. Solid lines give results for the dipole field obtained from Eq. (21a), while the dashed lines show corresponding results derived from the approximate solutions for the power law current sheet field given by Eq. (34a). The solid dots show spot values obtained from numerical integration using the full current sheet solution. For the case of the current sheet approximation the peak current depends only on $\Sigma_{P}^{*}$ and not on $\dot{M}$, so that only one dashed line is shown in plot (a), valid for all $\dot{M}$. The peak current in this case always occurs at the poleward boundary of the current sheet field lines, as indicated by the horizontal dotted lines at $\sim 14.95^{\circ}$ in plots (c) and (d).

proximation it is located consistently at the poleward boundary of the current sheet field lines at $\sim 14.95^{\circ}$, (dotted line) where the plasma angular velocity falls to zero. The numerically computed positions are located at a slightly higher colatitude, typically by $\sim 0.1^{\circ}$, like the total field-perpendicular current mentioned above. In practical application the peak current will thus be limited instead by the radial extent of the region to which the model is taken to apply, with the peak value occurring at its outer (poleward) boundary.

Figure 10 similarly provides results for the peak equatorial radial current, a parameter which relates directly to the magnitude of the azimuthal magnetic field outside of the current sheet (Fig. 1) $\left(B_{\varphi}(\mathrm{nT}) \approx 0.63 i_{\rho}\left(\mathrm{mA} \mathrm{m}^{-1}\right)\right)$. Figures 10a and $\mathrm{b}$ show that for the dipole field the peak current varies as $\Sigma_{P}^{* 1 / 2}$ and $\dot{M}^{1 / 2}$ (Eq. (21c)), while for the current sheet approximation it varies more strongly with the conductivity as $\Sigma_{P}^{* 1.71 / 2.71}$, and less strongly with the mass outflow rate as $\dot{M}^{1 / 2.71}$ (Eq. $34 \mathrm{c}$ ). The values given by the numerical integrations are in close agreement with the latter. The current sheet values are again higher than the dipole values by factors of $\sim 3$ to $\sim 5$ (typically $\sim 4$ ), for reasons given above. Fig- 

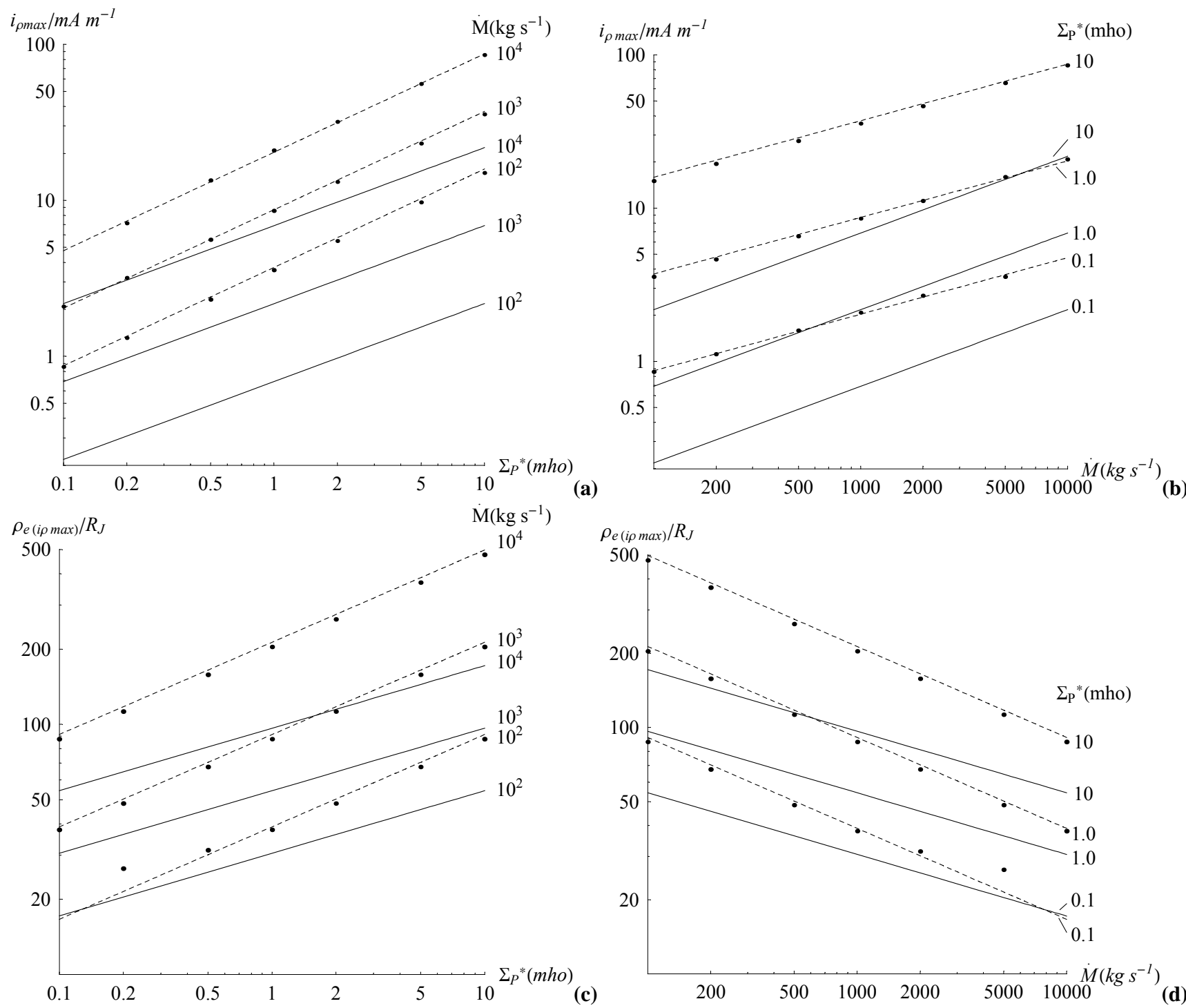

(a)

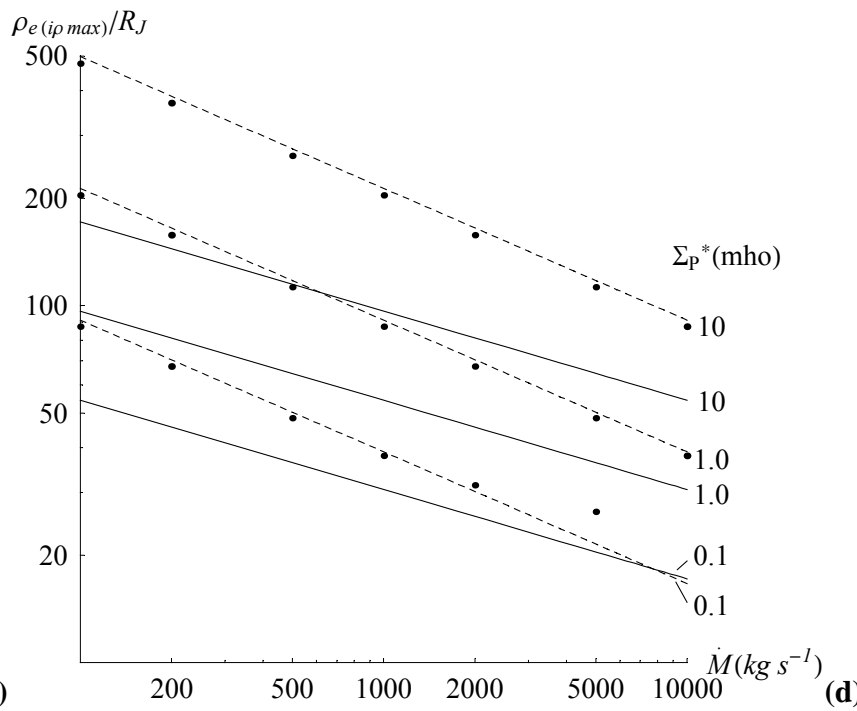

Fig. 10. Plots showing the magnitude and location of the peak sheet-integrated equatorial radial current intensity, and their dependence on $\Sigma_{P}^{*}$ and $\dot{M}$. Plot (a) shows the magnitude of the peak current versus $\Sigma_{P}^{*}$ in log-log format for $\dot{M}=100,1000$ and $10000 \mathrm{~kg} \mathrm{~s}{ }^{-1}$, while plot (c) shows the corresponding equatorial location of the peak in a similar format. Plots (b) and (d) similarly show the magnitude and location of the peak current versus $\dot{M}$ for $\Sigma_{P}^{*}=0.1,1$ and 10 mho. Solid lines give results for the dipole field obtained from Eq. (21c), while the dashed lines show corresponding results derived from the approximate solutions for the power law current sheet model given by Eq. (34c). The solid dots show spot values obtained from numerical integration of the full current sheet solution.

ures 10c and d show that the equatorial distance of the peak is typically located at $\sim 50 \mathrm{R}_{\mathrm{J}}$ for the dipole model, varying with the system parameters as $\Sigma_{P}^{* 1 / 4}$ and $\dot{M}^{-1 / 4}$, while for the current sheet approximation it is generally located at somewhat larger distances $\sim 90 \mathrm{R}_{\mathrm{J}}$, and varies more strongly as $\Sigma_{P}^{* 1 / 2.71}$ and $\dot{M}^{-1 / 2.71}$. The positions given by the numerical integrations are again in close agreement with the latter, except for small $\Sigma_{P}^{*}$ and large $\dot{M}$, where the position of the peak approaches $\rho_{e}^{*} \approx 21.78 \mathrm{R}_{\mathrm{J}}$. In fact for small $\Sigma_{P}^{*}$ and large $\dot{M}$, the peak current in the numerical solutions lies consistently at $\rho_{e}^{*}$, where the field models are joined. Such points are omitted from the plots. At large distances the ra- dial regime of applicability is again limited, such that the peak radial currents will actually occur at the outer boundary of the region for sufficiently large $\Sigma_{P}^{*}$ and/or sufficiently small $\dot{M}$, as can be determined from the position of the peak in Figs. 10c and d.

Results for the magnitude and location of the peak azimuth-integrated total equatorial radial current, equal, of course, to twice the peak azimuth-integrated total Pedersen current in each conjugate ionosphere, are shown in Fig. 11. Figures $11 \mathrm{a}$ and $\mathrm{b}$ show that the magnitude of the peak current for the dipole field increases with the conductivity as $\Sigma_{P}^{* 3 / 4}$, and less strongly with the mass outflow rate as $\dot{M}^{1 / 4}$ 

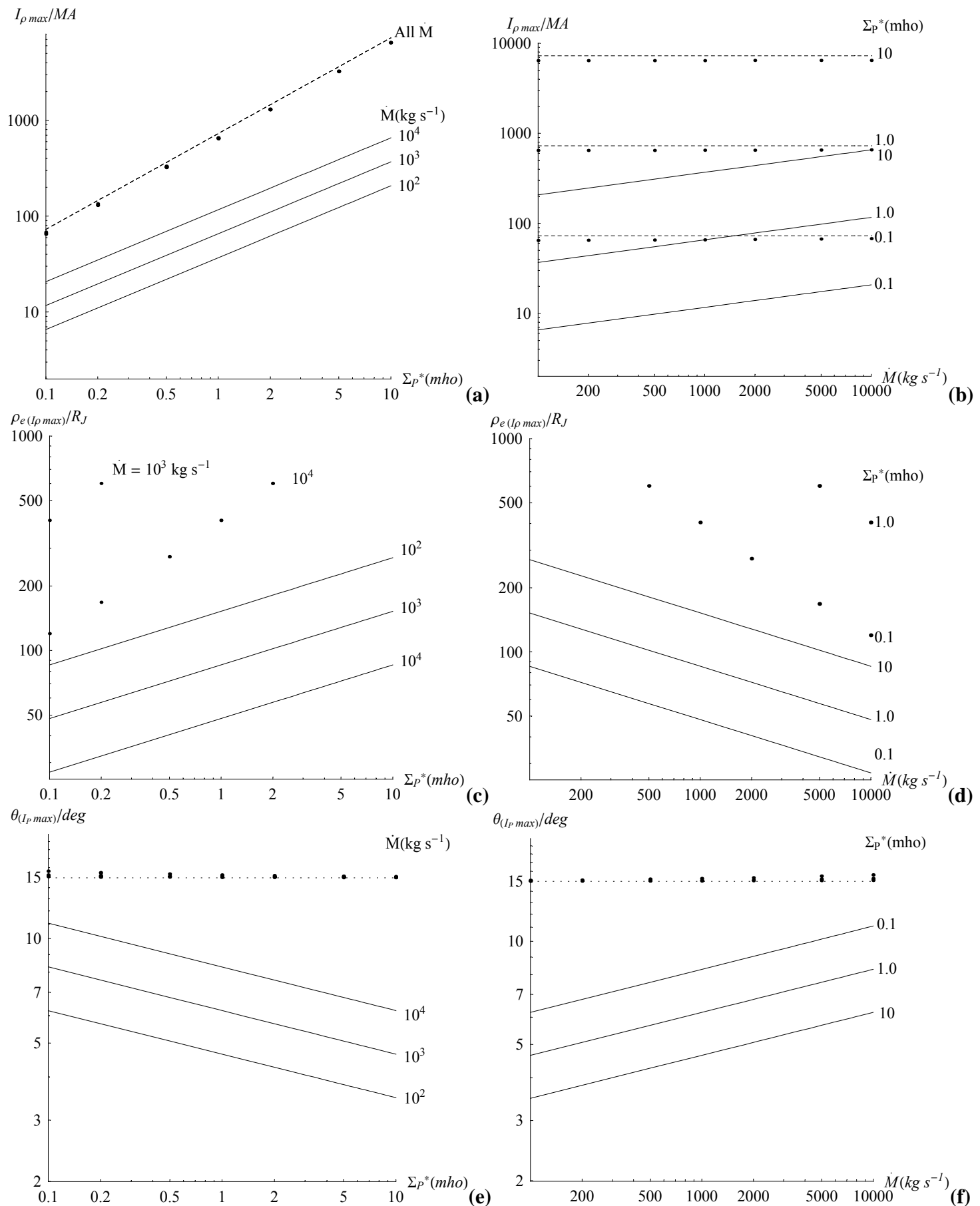

Fig. 11. Plots showing the magnitude and location of the peak azimuth-integrated total equatorial and ionospheric currents, and their dependence on $\Sigma_{P}^{*}$ and $\dot{M}$. Plot (a) shows the magnitude of the peak total equatorial radial current (equal to twice the peak total ionospheric Pedersen current) versus $\Sigma_{P}^{*}$ in $\log -\log$ format for $\dot{M}=100,1000$ and $10000 \mathrm{~kg} \mathrm{~s}^{-1}$, while plots (c) and (e) show the corresponding equatorial and ionospheric locations of the peak in a similar format. Plots (b), (d) and (f) similarly show the magnitude and location of the azimuth-integrated peak total current versus $\dot{M}$ for $\Sigma_{P}^{*}=0.1,1$ and $10 \mathrm{mho}$. Solid lines give results for the dipole field obtained from Eqs. ( $21 \mathrm{~b}, \mathrm{~d}$ ), while the dashed lines show corresponding results derived from the approximate solutions for the power law current sheet model given by Eqs. (34b, d). The peak total current in the latter model is independent of $\dot{M}$, so that only one dashed line is shown in (a). It occurs at infinity in the equatorial plane so that no dashed lines are shown in (c) and (d), or equivalently at the poleward boundary of current sheet field lines in the ionosphere at $\sim 14.95^{\circ}$ as shown in (e) and (f) (dotted line). The solid dots show spot values obtained from numerical integration of the full current sheet solution. In this case the peak values occur at large but finite distances such that only the closest of them are included in (c) and (d). 

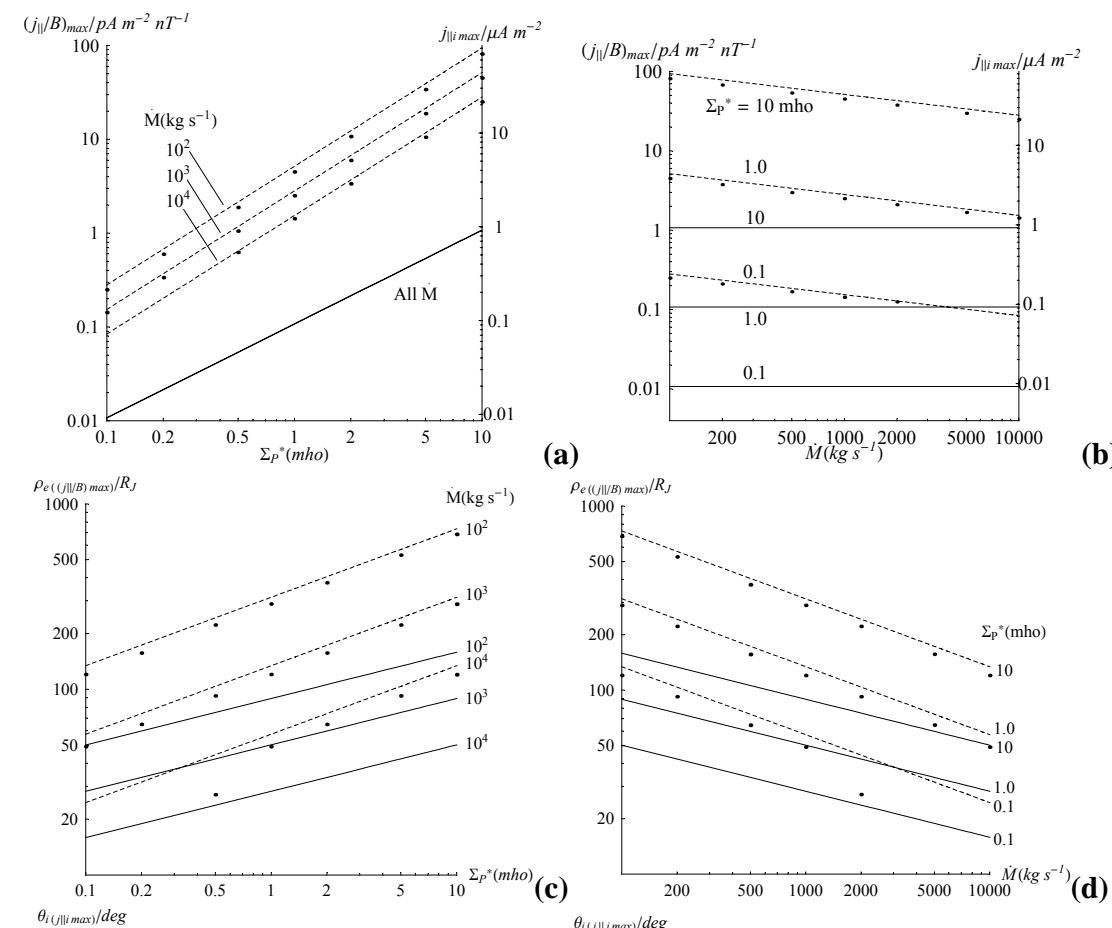

(b)
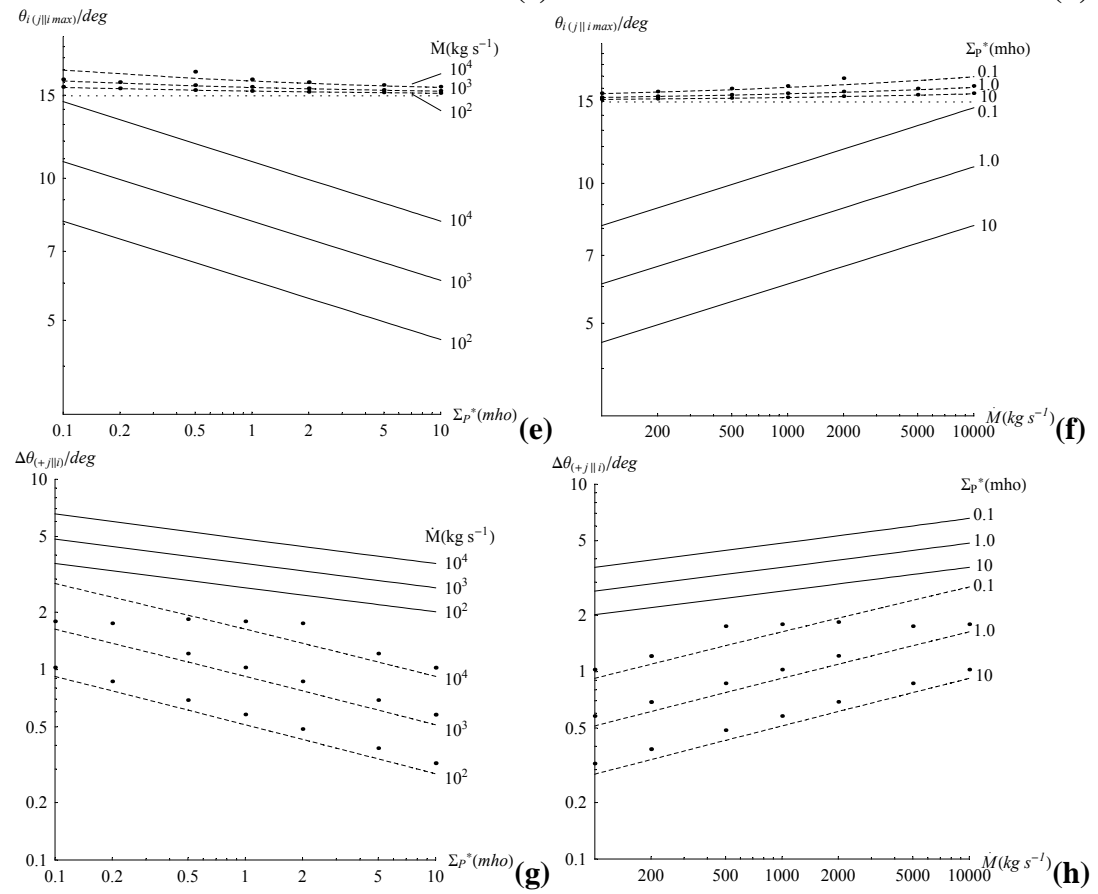

Fig. 12. Plots showing the magnitude, location and half-width of the peak upward-directed field-aligned current density, and their dependence on $\Sigma_{P}^{*}$ and $\dot{M}$. Plot (a) shows the magnitude of the peak total current density versus $\Sigma_{P}^{*}$ in $\log -\log$ format for $\dot{M}=100,1000$ and $10000 \mathrm{~kg} \mathrm{~s}^{-1}$, where the left-hand scale shows the peak current density in the ionosphere, while the right-hand scale shows the peak $\left(j_{\|} / B\right)$ value, simply related to the latter via Eq. (5b). Solid lines give results for the dipole field obtained from Eqs. (21e ,f), while the dashed lines show corresponding results derived from the approximate solutions for the power law current sheet model given by Eqs. (34e, f). The solid dots show spot values obtained from numerical integration of the full current sheet solution. Plot (c) shows the corresponding location of the peak $\left(j_{\|} / B\right)$ in the equatorial plane in a similar format, while plot (e) shows the conjugate location of the peak field-aligned current in the ionosphere. Plots (b), (d), and (f) similarly show the magnitude and equatorial and ionospheric locations of the peak current density versus $\dot{M}$ for $\Sigma_{P}^{*}=0.1,1$ and $10 \mathrm{mho}$. Plots $(\mathbf{g})$ and (h) show the latitudinal width of the upward field-aligned current region in the ionosphere in a similar format, defined as the full width at half maximum. 
(Eqs. 21b, d), while for the current sheet approximation it is linearly proportional to $\Sigma_{P}^{*}$ and independent of $\dot{M}$ (Eqs. $34 \mathrm{~b}$, d). The numerical values shown by the dots in the latter case are a little lower than the dashed line approximation for reasons given above for the Pedersen current. The value of the peak current is a factor of $\sim 5$ to $\sim 20$ (typically $\sim 10$ ) larger for the current sheet than for the dipole. The location of the peak in the equatorial plane (where the field-aligned current passes through zero), is shown in Figs. 11c and d. It is located typically at $\sim 90 \mathrm{R}_{\mathrm{J}}$ for the dipole field, scaling as $\Sigma_{P}^{* 1 / 4}$ and $\dot{M}^{-1 / 4}$, but occurs at infinity for the current sheet approximation (such that no dashed lines are shown in Figs. 11c and d), or, in other words, at the outer boundary of the relevant region in practical application. The peak value in the numerical curves, shown by the dots, occurs at large but finite radial distance, as mentioned above, typically well beyond the region of physical applicability $\left(\sim 500\right.$ to $\left.\sim 5000 \mathrm{R}_{\mathrm{J}}\right)$. Only the closest of them (for small $\Sigma_{P}^{*}$ and large $\dot{M}$ ) are included in Figs. 11c and d. The corresponding location of the peak azimuth-integrated Pedersen current in the conjugate ionosphere is shown in Figs. 11e and f. It is located typically at $\sim 6^{\circ}$ for the dipole field, scaling as $\Sigma_{P}^{*-1 / 8}$ and $\dot{M}^{1 / 8}$ as before, but for the current sheet it is located consistently at (for the approximation) or near (for the numerical values) the poleward boundary of the current sheet field lines at $\sim 14.95^{\circ}$ (dotted line).

Figure 12 shows results for the upward-directed fieldaligned current density, a parameter of relevance to the origins of the jovian auroras. The magnitude of the peak upward current is shown in Figs. 12a and $12 \mathrm{~b}$ in a similar format to the above, where, since $j_{\| i}$ and $\left(j_{\|} / B\right)$ are simply related through the constant factor $2 B_{J}$ in the approximation for the ionospheric magnetic field employed here (Eq. 5b), one plot serves the purpose of both parameters according to the leftand right-hand scales. These plots show that for the dipole field the peak upward current density depends linearly on $\Sigma_{P}^{*}$ and is independent of $\dot{M}$ (Eqs. 21e, f), while for the current sheet approximation it increases somewhat more rapidly with the conductivity as $\Sigma_{P}^{* 3.42 / 2.71}$ (i.e. as $\sim \Sigma_{P}^{* 1.26}$ ), while decreasing slowly with the mass outflow rate as $\dot{M}^{-0.71 / 2.71}$ (Eqs. 34e, f). The latter values agree well with those obtained from numerical integration, and exceed those obtained for the dipole field by factors of $\sim 10$ to $\sim 50$ (typically by $\sim 25)$. The position of the peak value of $\left(j_{\|} / B\right)$ in the equatorial plane is shown in Figs. $12 \mathrm{c}$ and d. It lies typically at distances of $\sim 50 \mathrm{R}_{\mathrm{J}}$ for the dipole field and varies as $\Sigma_{P}^{* 1 / 4}$ and $\dot{M}^{-1 / 4}$, while lying at larger typical distances beyond $\sim 100 \mathrm{R}_{\mathrm{J}}$ for the current sheet model and varies as $\Sigma_{P}^{* 1 / 2.71}$ and $\dot{M}^{-1 / 2.71}$. The position of the peak field-aligned current density in the ionosphere is shown in Figs. 12e and f. For the dipole it lies typically at a co-latitude of $\sim 8^{\circ}$ and scales in distance from the magnetic axis as $\Sigma_{P}^{*-1 / 8}$ and $\dot{M}^{1 / 8}$, while for the current sheet model it lies just equatorward of the boundary of current sheet field lines, with variations which are in the same sense as for the dipole, but with amplitudes which are much smaller. In Figs. $12 \mathrm{~g}$ and $\mathrm{h}$ we finally show a measure of the latitudinal width of the region of upward fieldaligned current, potentially related to the latitudinal width of associated jovian auroras, plotted versus $\Sigma_{P}^{*}$ and $\dot{M}$, respectively. The width of the upward current given here is the full width at half maximum. The solid and dashed lines show results for the dipole and power law current sheet approximation, respectively. The results were derived from the fact that in the equatorial plane the value of $\left(j_{\|} / B\right)$ reaches half its peak positive value for the dipole field at normalised radial distances $\left(\rho_{e} / R_{D e}\right)$ of $\sim 0.629$ and $\sim 1.470$ (see Fig. 3e), while for the power law current sheet approximation the corresponding values of $\left(\rho_{e} / R_{C S e}\right)$ are $\sim 0.940$ and $\sim 8.337$ (see Fig. 7e). The dots again show spot values obtained numerically using the full current sheet field. It can be seen that the width for the dipole field is typically $\sim 3^{\circ}-5^{\circ}$, decreasing modestly with increasing $\Sigma_{P}^{*}$ and increasing modestly with increasing $\dot{M}$ (as $\Sigma_{P}^{*-1 / 8}$ and $\dot{M}^{1 / 8}$, respectively). For the current sheet model the thickness is reduced to $\sim 0.5^{\circ}-1.5^{\circ}$ (less if the system is limited in radial distance), varying in the above manner more strongly with the system parameters (as $\sim \Sigma_{P}^{*-0.26}$ and $\sim \dot{M}^{0.26}$ ).

\section{Summary}

In this paper we have considered the steady-state properties of the magnetosphere-ionosphere coupling current system that flows in Jupiter's middle magnetosphere, associated with the enforcement of partial corotation on outwardflowing plasma from the Io torus. The solutions depend on the values of two parameters, the effective Pedersen conductivity of the jovian ionosphere $\Sigma_{P}^{*}$, and the mass outflow rate of iogenic plasma $\dot{M}$, these being taken to be constants. However, their values remain uncertain at present, thus prompting the study presented here of how the solutions depend on these parameters over wide ranges of the latter. We have also focussed on two models of the magnetospheric poloidal field, taken for simplicity to be axisymmetric. The first is the planetary dipole alone, which constitutes an instructive paradigm. Some general results for this case have previously been given by Hill $(1979,2001)$. Here we have provided a complete analytic solution for this case, showing how the plasma angular velocity and current components scale in space and in amplitude with $\Sigma_{P}^{*}$ and $\dot{M}$. We find that the plasma angular velocity and current components scale in equatorial radial distance as $\left(\Sigma_{P}^{*} / \dot{M}\right)^{1 / 4}$, as found previously by Hill (and correspondingly as $\left(\Sigma_{P}^{*} / \dot{M}\right)^{1 / 8}$ ) in the ionosphere), while each current component scales in amplitude as $\left(\Sigma_{P}^{*(1+\gamma) / 2} \dot{M}^{(1-\gamma) / 2}\right.$, where $\gamma$ has a particular value for each component. The scales in space and amplitude then combine to produce current values which depend only on $\dot{M}$ at a fixed position at small radial distances, and only on $\Sigma_{P}^{*}$ at a fixed position at large radial distances, these dependencies then requiring current variations at small and large distances 
with particular powers of the distance, as $\rho_{e}^{2(1+\gamma)}$ at small distances, and as $\rho_{e}^{-2(1-\gamma)}$ at large distances.

These results provide useful background for the second, more realistic field model, based on Voyager data, in which the equatorial field strength is significantly less than for the dipole field due to the radial distension of the middle magnetosphere field lines, and is taken to vary with distance as a power law $\rho_{e}^{-m}$. Solutions for a few spot values of $\Sigma_{P}^{*}$ and $\dot{M}$ have previously been presented by Cowley et al. (2002, 2003), obtained by numerical integration of the corresponding Hill-Pontius equation. Here we have derived an analytic approximation, applicable to the power law regime, which shows how the plasma angular velocity and current components scale with $\Sigma_{P}^{*}$ and $\dot{M}$ in this case. We find that these solutions provide accurate approximations to the full numerical results within the power law regime (roughly $\rho_{e}>20 \mathrm{R}_{\mathrm{J}}$ ) over very wide ranges of the system parameters, provided $\left(\Sigma_{P}^{*} / \dot{M}\right)$ is not too small $\left(\sim 10^{-4}\right.$ mho skg $^{-1}$ or larger $)$. The results show that the conclusions concerning the nature of the current sheet solutions, and their relation to the dipole solutions, which were drawn previously on the basis of a limited number of numerical investigations are generally valid over wide ranges of the parameters. In particular, it has been shown that in the current sheet model the field-aligned current flows unidirectionally outward from the ionosphere into the current sheet over the whole current sheet, in all cases of interest. The closure of this current must then occur on field lines at higher latitudes which map to the outer magnetosphere and tail, which are not described by the present theory. This situation contrasts with the dipole model, in which (at least in principle) all the flux in the system is described by the theory, such that complete current closure occurs between the equator and the pole. The results for the power law current sheet show that the plasma angular velocity and currents now scale in radial distance as $\left(\Sigma_{P}^{*} / \dot{M}\right)^{1 / m}$, while each current component again scales in amplitude as $\Sigma_{P}^{*(1+\gamma) / 2} \dot{M}^{(1-\gamma) / 2}$, where the values of $\gamma$ for each component exceed those of the corresponding component for the dipole field (at least for $m>2$ as considered here). The current components thus scale as a somewhat higher power of $\Sigma_{P}^{*}$ for the current sheet than for the dipole, and as a somewhat lower power of $\dot{M}$. These scales in space and amplitude again combine to produce current values which depend only on $\dot{M}$ at a fixed position at small radial distances, and only on at a fixed position at large radial distances (both being general properties of the solutions), these dependencies then requiring current variations as $\rho_{e}^{m(1+\gamma) / 2}$ at small distances, and as $\rho_{e}^{-m(1-\gamma) / 2}$ at large distances. The absolute values of the currents are also higher for the current sheet model than for the dipole, by a factor of $\sim 4$ for the Pedersen and equatorial currents, $\sim 10$ for the total current flowing in the circuit, and $\sim 25$ for the field-aligned current densities. These factors do not vary greatly over the range of system parameters considered here.

Acknowledgements. JDN was supported during the course of this study by a PPARC Quota Studentship, and SWHC by PPARC Senior Fellowship PPA/N/S/2000/00197.

Topical Editor T. Pulkkinen thanks V. M. Vasyliunas and another referee for their help in evaluating this paper.

\section{References}

Bagenal, F.: Empirical model of the Io plasma torus, J. Geophys. Res., 99, $11043,1994$.

Bagenal, F.: The ionization source near Io from Galileo wake data, Geophys. Res. Lett., 24, 2111, 1997.

Belcher, J. W.: The low-energy plasma in the jovian magnetosphere, in: Physics of the Jovian Magnetosphere, (Ed) Dessler, A. J., Cambridge Univ. Press, Cambridge, UK, 68, 1983.

Broadfoot, A. L., Sandel, B. R., Shemansky, D. E., McConnell, J. C., Smith, G. R., Holberg, J. B., Atreya, S. K., Donahue, T. M., Strobel, D. F., and Bertaux, J. L.: Overview of the Voyager ultraviolet spectrometry results through Jupiter encounter, J. Geophys. Res., 86, 8259, 1981.

Bunce, E. J. and Cowley, S. W. H.: Divergence of the equatorial current in the dawn sector of Jupiter's magnetosphere: analysis of Pioneer and Voyager magnetic field data, Planet. Space Sci., 49, 1089, 2001.

Connerney, J. E. P., Acuña, M. H., and Ness, N. F.: Modeling the Jovian current sheet and inner magnetosphere, J. Geophys. Res., 86, 8370, 1981.

Connerney, J .E. P., Acuña, M. H., Ness, N. F., and Satoh, T.: New models of Jupiter's magnetic field constrained by the Io flux tube footprint, J. Geophys. Res., 103, 11 929, 1998.

Cowley, S. W. H. and Bunce, E. J.: Origin of the main auroral oval in Jupiter's coupled magnetosphere-ionosphere system, Planet. Space Sci., 49, 1067, 2001.

Cowley, S. W. H. and Bunce, E. J.: Modulation of jovian middle magnetosphere currents and auroral precipitation by solar windinduced compressions and expansions of the magnetosphere: Initial conditions and steady state, Planet. Space Sci., 51, 31-56, 2003.

Cowley, S. W. H., Nichols, J. D., and Bunce E. J.: Steady-state distributions of flow, current, and auroral precipitation in Jupiter's middle magnetosphere: Solutions for current sheet and dipole magnetic field models, Planet. Space Sci., 50, 717, 2002.

Cowley, S. W. H., Bunce, E. J., and Nichols, J. D.: Origins of Jupiter's main oval auroral emissions, J. Geophys. Res., 108,(A4), 8002, doi: 10.1029/2002JA009329, 2003.

Edwards, T. M., Bunce, E. J., and Cowley, S. W. H.: A note on the vector potential of Connerney et al.'s model of the equatorial current sheet in Jupiter's magnetosphere, Planet. Space Sci., 49, $1115,2001$.

Hill, T. W.: Inertial limit on corotation, J. Geophys. Res., 84, 6554, 1979.

Hill, T. W.: The jovian auroral oval, J. Geophys. Res., 106, 8101, 2001.

Hill, T. W., Dessler, A. J., and Goertz, C. K.: Magnetospheric models, in: Physics of the Jovian Magnetosphere, (Ed) Dessler, A. J., Cambridge Univ. Press, Cambridge, UK, 353, 1983.

Huang, T. S. and Hill, T. W.: Corotation lag of the jovian atmosphere, ionosphere and magnetosphere, J. Geophys. Res., 94, 3761, 1989.

Khurana, K.K.: Influence of solar wind on Jupiter's magnetosphere deduced from currents in the equatorial plane, J. Geophys. Res., 106, $25999,2001$. 
Khurana, K. K. and Kivelson, M. G.: Inference of the angular velocity of plasma in the jovian magnetosphere from the sweepback of magnetic field, J. Geophys. Res., 98, 67, 1993.

Pontius Jr., D. H.: Radial mass transport and rotational dynamics, J. Geophys. Res., 102, 7137, 1997.

Siscoe, G. L. and Summers, D.: Centrifugally-driven diffusion of iogenic plasma, J. Geophys. Res., 86, 8471, 1981.

Southwood, D. J. and Kivelson, M. G.: A new perspective con- cerning the influence of the solar wind on Jupiter, J. Geophys. Res., 106, 6123, 2001.

Strobel, D. F. and Atreya, S. K.: Ionosphere, in: Physics of the Jovian Magnetosphere, (Ed) Dessler, A. J.,Cambridge Univ. Press, Cambridge, UK, 51, 1983.

Vasyliunas, V. M.: Plasma distribution and flow, in: Physics of the Jovian Magnetosphere, (Ed) Dessler, A. J., Cambridge Univ. Press, Cambridge, U.K., 395, 1983. 